\title{
DIE VÖLKERRECHTLICHEN VERTRÄGE DER VEREINIGTEN STAATEN VON AMERIKA ÜBER FRAGEN DER STAATSANGEHÖRIGKEIT EINSCHLIESSLICH EINBÜRGERUNG UND WEHRPFLICHT ${ }^{1}$
}

\author{
Von Hellmuth Hecker und Knud Krakau
}

I.

Die Vereinigten Staaten verdanken buchstäblich ihre Entstehung und einen guten Teil ihrer raschen Entwicklung zur ersten Industrie- und Militärmacht der Erde einem stetigen Strom von Einwanderern. Das hieraus zunächst erwachsende praktische und vitale Interessse an einer gut hundert Jahre geübten liberalen Einwanderungspolitik fand alsbald auch seine ideologische Überhöhung: die Öffnung des Landes trug nach amerikanischem Selbstverständnis nicht nur den Bedürfnissen der eigenen Entwicklung Rechnung, sondern hatte darüber hinaus eine ganz unbedingte politisch-moralische Qualität. Die Entstehung und das erstaunliche Wachstum der Vereinigten Staaten erschienen dieser Interpretation nicht als beliebige Ereignisse, sondern anfänglich als Vollendung eines göttlichen Heilsplanes, sodann in einem säkularisierten (quasi-Hegelianischen) Sinne als gesteigerte Verwirklichung überhaupt möglicher politisch-moralischer Entwicklung, ja als Vollendung von Geschichtlichkeit schlechthin. In diesem Sinne sind etwa folgende Formulierungen Alexander Hamiltons in der ersten Nummer des Federalist zu verstehen: „... it seems to have been reserved to the people of this country . . . to decide the important question whether societies of men are really capable or not of establishing good government from reflection and choice, or whether they are forever destined to depend for their political constitutions on accident and force". Die gegenwärtige - d. h. damalige, immer nach Hamilton - Krise verlange eine Entscheidung, „and a wrong election of the part we shall act may ... deserve to be considered as the general misfortune of mankind". Was für Hamilton noch (rhetorische?) Frage schien, war für Zeitgenossen und vor allem Nachfolger Gewißheit: Amerika hat te richtig entschieden. Daraus folgte: Amerika mußte nicht nur den Prototyp politischer Organisation selbst verwirklichen, und zwar u.a. mit Hilfe der Einwanderer; daraus resultiert das praktische eigene Bedürfnis für sie. Die historisch hervorgehobene Rolle beinhaltete aber zugleich auch Verpflichtung und Verantwortung: für die weniger Begünstigten, noch vom Dunkel der alten (feudalen) unfreien Verhältnisse umfangenen Menschen der (damaligen) Alten Welt das Licht der Freiheit sichtbar zu machen und den Mühseligen, Beladenen und Unterdrückten in aller Welt Hafen und Heimat zu bieten². Die bloße Ankunft hier verhieß Befreiung aus den Banden Status-bedingter Unfreiheiten. Diese und

1 Siehe dazu im Dokumententeil am Schluß die inhaltlichen, alphabetischen und chronologischen Ubersichten.

2 Vgl. mit Nachweisen K. Krakau, Missionsbewußtsein und Völkerrechtsdoktrin in den Vereinigten Staaten (Frankfurt/M. und Berlin 1967), S. 73, 116; oder etwa R. B. Nye, This almost chosen people (Michigan State Univ. Press 1966); G. M. Lyons ed., America. Purpose and power (Chicago 1965). 
ähnliche ideologische Symbole begleiten bis heute die Diskussion um die Einwanderungspolitik $^{3}$ und die damit zusammenhängenden Staatsangehörigkeits-Fragen.

Das praktische eigene Bedürfnis nach Einwanderern ist dagegen schon seit längerem in den Hintergrund getreten. Nach den großen Einwanderungswellen in der Mitte und zweiten Hälfte des 19. Jahrhunderts rückten die Vereinigten Staaten im Zusammenhang mit der wirtschaftlichen Rezession der 1880er und 1890er Jahre deutlich von ihrer früheren liberalen Einwanderungspolitik ab. Diese Wendung richtete sich zuerst gegen die Chinesen ${ }^{4}$, später gegen die Japaner, führte 1917 zur "asiatischen Sperrzone“ und kulminierte 1921 schließlich in dem nationalen Quotensystem. Dieses Einwanderungsgesetz bevorzugte die klassischen Einwanderungsländer Westeuropas, indem es die damaligen relativen Anteile dieser Herkunftsländer an der Gesamtbevölkerung der USA zum Maßstabe für die Quoten setzte, nach denen künftighin die zulässigen Einwandererzahlen für jedes Land errechnet werden sollten. Man ging stillschweigend davon aus, daß die damalige ethnische Mischung der amerikanischen Bevölkerung gewissermaßen einen Idealzustand erreicht habe, der nur noch fortgeschrieben, d. h. an Gesamtzahl zwar vermehrt, aber nicht mehr im Mischungsverhältnis geändert werden dürfe. Nun handelten die Vereinigten Staaten unter Verdrängung der Einwanderungsideologie so, wie vermutlich jeder Staat in vergleichbarer Lage handeln würde. Sie reduzierten die Einwanderungsmöglichkeiten, weil sie ihre Aufnahmekapazität in dem Sinne für begrenzt erachteten, daß sie in weiterer uneingeschränkter Einwanderung eine Gefährdung des sozialen und ökonomischen Status der eigenen Bevölkerung erblickten. Dabei spielte die rassische Aversion gegen nichtweiße Einwanderer eine besondere Rolle, die wiederum nicht unwesentlich darauf zurückzuführen ist, daß sich in ihr die Status-Angst gegenüber extrem ("unfair") billigen — insbesondere asiatischen - Arbeitskräften artikulierte.

Gewisse Schwierigkeiten bereitete dabei der Widerspruch zur früheren Einwanderungsideologie. Aber er war einmal dialektisch aufzulösen: Der Sache der Freiheit für die Welt wurde am wenigsten dadurch gedient, daß diejenigen, die diese Freiheit schon verwirklicht hatten, deren ökonomische Grundlagen unterminierten. Vielmehr mußten diese Grundlagen im Interesse eben der Wirkungskraft dieser Freiheit für die Welt gesichert werden, und sei es durch die zeitweilige Drosselung der Einwanderung.

Zum anderen aber wurde in einem Lande, das auf Einwanderer nicht mehr wie früher angewiesen ist - immerhin aber macht heute die Einwanderung bei sinkenden Geburtenziffern rund 20 Prozent des gesamten Bevölkerungszuwachses aus -, ein langwährender Kampf um eine erneute Liberalisierung der Einwanderungspolitik in erheblichem Umfange mit eben dem klassischen ideologischen Argument geführt. Im Vergleich dazu wirkt der hierbei ebenfalls häufig verwendete Hinweis auf "needed skills" eher wie die unumgängliche Vorbeugung vor dem nun einmal zugleich pragmatischen Sinn der Amerikaner. Diese Bemühungen waren besonders nach 1945 - nicht während des Krieges - mit mehreren Sondergesetzen zugun-

3 Vgl. Außerungen Präs. Johnsons und von A. P. Schwartz vom Department of State: Foreign and domestic implications of U. S. immigration laws, im Zusammenhang mit der Reform des Einwanderungsgesetzes, am 13. 1. bzw. 3. 4. 1964, Dpt. of St. Bull. 50 (1964 I), S. 211 bzw. 675, $676 \mathrm{f}$.

4 Vgl. den Vertrag vom 17. 11. 1880 mit China, in dem China sich mit einer im Interesse der Vereinigten Staaten von diesen etwa vorzunehmenden Beschränkung (nicht dem vollen Verbot) der Einwanderung chinesischer Arbeiter einverstanden erklärte, Malloy, S. 237, und den Vertrag vom 17. 3. 1894, Art. I und IV, Malloy, S. 241, der für die Dauer von 10 Jahren jede Einreise von chinesischen Arbeitern völlig untersagte und den legal sich in den USA aufhaltenden Chinesen hinsichtlich ihrer persönlichen Rechte die Meistbegünstigung einräumte, davon aber die Naturalisierung ausschloß. 
sten verschiedener Gruppen von Flüchtlingen, Verfolgten usw. zunächst punktuell erfolgreich und führten schließlich dazu, daß das Einwanderungsgesetz von 1965 das willkürliche nationale Quotensystem bis 1968 stufenweise abbaute und durch eine Reihe sachlicher Auswahlkriterien (Familieneinheit, Flüchtlingsstatus, berufliche Qualifikation) innerhalb bestimmter Höchstgrenzen ersetzte ${ }^{5}$. Diese Limitierung knüpft nicht mehr an einzelne Nationen, sondern an Hemisphären an: jährlich 170000 Einwanderer für die östliche (Europa, Afrika, Asien; maximal 20000 für jedes einzelne Land) und 120000 für die westliche Hemisphäre (ohne die 20000Grenze). Das neue System bewirkte sofort eine erhebliche Umleitung der Einwandererströme: Das Schwergewicht verlagerte sich von den „klassischen“ Herkunftsländern (Nord- und Westeuropa) mit früher hohen Quoten auf Staaten einschließlich solcher mit nicht weißer Bevölkerung -, die nach dem alten System mit niedrigen Quoten benachteiligt gewesen waren (Philippinen, Japan, Süd- und Südosteuropa, auch Afrika, Westindien) ${ }^{6}$.

Die Praxis der besonders vor Ende des 19. Jahrhunderts kaum gehinderten Einwanderung warf, unabhängig vom Motiv für diese Haltung, konkrete Probleme hinsichtlich ihrer staatsangehörigkeitsrechtlichen Folgen auf, die eine Regelung verlangten, wenn die zugrundeliegende Politik und das Verhältnis zu den Heimatländern der amerikanischen Neu-Bürger nicht gestört werden sollten. Dieser Umstand erklärt, daß die Vereinigten Staaten im Laufe der Zeit zahlreiche staatsangehörigkeitsregelnde bilaterale Verträge ${ }^{7}$ abgeschlossen haben, andererseits an nur vier multilateralen Vertragswerken ${ }^{3}$ beteiligt sind, letzteres eben deshalb, weil diese zur Bewältigung der spezifischen amerikanischen Interessenlagen wenig beitragen.

II.

Unter den bilateralen Verträgen sei vorweg kurz die verwandte, aber ganz unspezifische Gruppe von vertraglichen Staatsangehörigkeits-Regelungen erwähnt, die mit der materiellen Friedensregelung nach dem Revolutions- oder Unabhängigkeitskrieg (mit Großbritannien, formell schon Freundschafts-, Handels- und Schifffahrtsvertrag genannt, dem sogenannten Jay-Vertrag von 1794) sowie mit späteren Gebietserwerbungen (Frankreich, Spanien, Mexiko, Rußland, Dänemark) ${ }^{9}$ zusammenhängen. Es handelt sich um Verfügungen über die Staatsangehörigkeit und

5 Einwanderungsgesetz vom 3. 10. 1965, Public Law 89-236, Stat. Bd. 79, S. 916 ff., U. S. C. Title 8, Ch. 12, SS $1101 \mathrm{ff}$. Für die nachfolgend erw. Limitierungen s. bes. \S 1101 (a) (27) (A), 1151 (a), 1152 (a), und sec. 21 (e) in der Formulierung des Pub. L. 89-236. Vgl. im übrigen die Ubersicht zur Geschichte der amerikanischen Einwanderungspolitik bei A. P. Schwartz, a. a. O., S. 677 ff.; für die ältere Zeit ferner L. Stoddard, Re-forging America. The story of our nationhood (London 1927); A. Spitzl, Die Einwanderung nach den Vereinigten Staaten von Nordamerika (sic), eine staats- und völkerrechtliche Untersuchung (Diss. Frankfurt 1931).

6 Die alte Quote der Philippinen betrug z. B. bis 1965 nur 100; das Haushaltsjahr (bis 30. 6.) 1969 sah 25425 philipp. Einwanderer; Italien stieg von 10344 (1965) auf 24465 (1969, über die generelle Höchstgrenze hinaus). Umgekehrt sanken die entsprechenden Zahlen z. B. für Canada von 40013 auf 12263 (1970) oder für Irland von 5563 auf etwas über 1000. Vgl. den auf soeben veröffentlichten Statistiken beruhenden Bericht von F. Belair: „1965 immigration law molds different U. S. ,melting pot' “, in: Internat. Herald Tribune vom 1. 9. 1970. "Dennoch gibt es auch jetzt wieder für manche Länder - z. B. Mexiko lange Wartelisten; neue Reformen werden erwogen. Vgl. auch neue Daten des Amtes für Bevölkerungsstatistik über die im wesentlichen durch das Ges. von 1965 ermöglichte etwa vervierfachte (rund 1/2 Mill.) Einwanderung von Farbigen aus Afrika, Mittel- und Südamerika, China, Japan und Asien allgemein, nach Internat. Herald Tribune vom 31.12. 1970.

7 Ausführlich dazu Hackworth, S. 1-434; Whiteman, S. 79 ff., $560 \mathrm{ff}$. (vgl. Abkürzungen a. E.)

8 Interamerik. Abkommen über den Status Eingebürgerter v. 13. 8. 1906 und über die Staatsangehörigkeit der Ehefrau v. 26. 12. 1933 sowie das Haager Protokoll über Staatsangehörigkeit und Wehrdienst v. 12. 4. 1930. Näheres über Quellen usw. hierzu: H. Hecker, Mehrseitige völkerrechtliche Verträge zum Staatsangehörigkeits-Recht (Frankfurt/M. und Berlin 1970, SGS Bd. 30).

9 Die nachfolgende Erwähnung einzelner Staaten mit Jahreszahl bedeutet Vertrag der USA mit . . und Jahr des Abschlusses. Nähere Daten und Fundorte für sämtliche hier behandelten Verträge finden sich in dem dokumentarischen Teil unten S. $82 \mathrm{ff}$. 
über Staatsangehörige, wie sie typischerweise bei derartigen Anlässen getroffen werden. Erwähnt seien nur die außer bei den Louisiana- und Florida-Transaktionen stets (auch schon im Jay-Vertrag) vorgesehenen Optionsmöglichkeiten für die betroffenen Bevölkerungen. Im übrigen bieten diese Verträge keine Besonderheiten.

\section{III.}

Hiervon abgesehen gibt es zwei Typen von vertraglichen StaatsangehörigkeitsRegelungen. Der erste hat unmittelbar und ausschließlich die Regelung der Staatsangehörigkeits-Konflikte zum Gegenstand, die sich aus der Konkurrenz der Staatsangehörigkeit des Heimat- und des Einwanderungslandes ergeben. Der zweite Typ regelt andere Materien, insbesondere den Wehrdienst, und zeitigt dadurch mittelbare Wirkungen für die Staatsangehörigkeit.

Hier sei zunächst der Einwanderungs- und Staatsangehörigkeits-Vertrag im engeren Sinne analysiert. Konflikte ergaben sich, als sich im Laufe des 19. Jahrhunderts die wirtschaftlichen und politischen Kontakte mit den Heimatländern der Einwanderer mehrten. Die Frage stellt sich: Wer erkennt wessen Staatsangehörigkeit an, wenn die Vereinigten Staaten den Einwanderer naturalisieren? Der heute selbstverständliche Grundsatz, daß jeder Staat nur seine eigene Staatsangehörigkeit regeln kann, diese Regelung aber, von wenigen Grenzfällen abgesehen, von dritten Staaten auch anerkannt werden muß, war noch keineswegs fest verankert. Hinzu kam das Problem der Rückwanderung. Viele Neuankömmlinge kamen von vornherein nur für vorübergehende $Z$ wecke nach Amerika: als Abenteurer, um schnell reich $\mathrm{zu}$ werden, um dem Wehrdienst oder einer Strafe in ihrem Heimatland zu entgehen. Viele kehrten nach Verwirklichung ihres Zweckes oder wegen der Unmöglichkeit, ihn zu realisieren, oder aus sonstigen Gründen in ihre alte Heimat zurück. Welche Staatsangehörigkeit oder Staatsangehörigkeiten besaßen sie, wenn sie in Amerika naturalisiert worden waren? Konnten vor der Auswanderung begangene Taten oder die Auswanderung als solche strafrechtlich verfolgt, der Wehrdienst nachgefordert oder sein Versäumnis bestraft werden? Ist der alte (z. B. europäische) Heimatstaat - von seinem inneren Recht abgesehen - dem neuen Heimatstaat USA gegenüber völkerrechtlich gehindert, in der angedeuteten Weise gegen den Rückkehrer vorzugehen? Kann, muß er ihn wieder aufnehmen?

Dieser Problemkreis wurde nach der Einwanderungswelle in der Mitte des vorigen Jahrhunderts für regelungsbedürftig erachtet. Der Historiker und Diplomat George Bancroft handelte im Jahre 1868 mit dem Norddeutschen Bund sowie vier süddeutschen Staaten (Baden, Bayern, Hessen, Württemberg) eine Reihe von Verträgen aus, die - inhaltlich fast identisch, mit gewissen Abweichungen im Falle Badens diese Fragen erstmalig vertraglich zu regeln suchten (sog. Bancroft-Verträge im engeren Sinne). Die Tatsache, daß diese Verträge dem Wortlaut nach vollkommen wechselseitige Wirkungen entfalten sollten, darf dabei nicht darüber hinwegtäuschen, daß es sich tatsächlich um die Regelung überwiegend einseitiger Verhältnisse - nämlich die Migration von Europa nach Amerika nebst einem gewissen Rückstrom - handelte. Die Auswanderung von Amerika nach Hessen-Darmstadt etwa und Rückkehr nach Amerika war gewiß nicht sehr bedeutend. - Nach diesem Vorbild wurden später noch ähnliche Verträge mit zahlreichen anderen Staaten geschlossen. Man kann sie als Bancroft-Verträge im weiteren Sinne bezeichnen. Sie werden auch Einbürgerungs- und Naturalisationsverträge genannt. 
Die ursprünglichen Bancroft-Verträge regeln - wobei das Verhältnis BayernUSA als Beispiel diene und ferner die vollkommene Wechselseitigkeit zu berücksichtigen ist - übereinstimmend folgende Punkte:

1. Bayern erkennt bei fünfjährigem Aufenthalt eines Bayern in den Vereinigten Staaten dessen dort durch Naturalisation erworbene Staatsangehörigkeit an und behandelt ihn entsprechend. 2. Die Rückwanderung des in Amerika naturalisierten Bayern in sein Herkunftsland Bayern ohne die Absicht, nach Amerika zurückzukehren - wobei das Fehlen einer solchen Absicht nach zwei Jahren widerlegbar vermutet wird - , soll als Verzicht auf die durch Naturalisation erworbene amerikanische Staatsangehörigkeit behandelt werden. 3. Strafverfolgung von Taten, die vor der ersten Auswanderung von Bayern nach Amerika begangen worden sind, ist auch nach der Rückkehr nach Bayern zulässig.

Staatsangehörigkeits-Konflikte waren aber damit noch immer nicht aus der Welt geschafft. Gesichert war nur die Anerkennung der durch Naturalisation erworbenen (amerikanischen) Staatsangehörigkeit durch den Heimatstaat des Auswanderers (Bayern) und des stillschweigenden Verzichtes auf diese (amerikanische) Staatsangehörigkeit durch den Naturalisierungsstaat (USA) im Falle der Rückwanderung. Unklar blieb das Schicksal der ursprünglichen (bayerischen) Staatsangehörigkeit des Auswanderers. Die Verträge selbst ließen offen, ob sie bezweckten: Verlust dieser (bayerischen) Staatsangehörigkeit, bloße Anerkennung der (amerikanischen) Naturalisations-Staatsangehörigkeit mit der Folge der Doppelstaatlichkeit oder schließlich Doppelstaatigkeit mit Vorrang der neuen (amerikanischen) Staatsangehörigkeit. Die durch die Verträge geschaffene Rechtslage war durch die Staatsangehörigkeits-Regelungen der deutschen Vertragsstaaten zu ergänzen. Diese waren uneinheitlich, so daß je nach Rechtslage die eine oder andere der erwähnten Folgen eintrat und die Verträge insoweit ihren Zweck verfehlten. Erst die reichseinheitliche Regelung der Staatsangehörigkeit von 1870/71 enthielt als ausdrücklichen und echten Verlustgrund auch den in den Bancroft-Verträgen ins Auge gefaßten Tatbestand ${ }^{10}$.

Das spricht zugleich dafür, daß die Bancroft-Verträge die Doppel-Staatsangehörigkeit vermeiden und $\mathrm{n} u \mathrm{r}$ die jeweils neu erworbene Staatsangehörigkeit gelten lassen wollten, also eine unmittelbare Regelung des Verlustes der alten (Herkunfts-) Staatsangehörigkeit beabsichtigten. Für diese Interpretation streitet auch die inhaltliche Gestaltung des zweiten Problems der Bancroft-Verträge, der Rückwanderung. Einmal ist in diesem letzten Zusammenhange von dem Verzicht auf die durch Naturalisation erworbene Staatsangehörigkeit die Rede. Zum anderen können die Rückwanderer die Staatsangehörigkeit ihres früheren Heimatstaates nach dessen allgemeinen Gesetzen lediglich $\mathrm{n}$ e $\mathrm{u}$ erwerben, mithin müssen sie sie durch die qualifizierte Naturalisation in den Vereinigten Staaten nach der Absicht der BancroftVerträge verloren haben. - Von dem gelegentlichen Verzicht auf die Fünf-JahresFrist oder der Verweisung auf andere innergesetzliche Fristen (Ecuador) abgesehen, veranlaßte dieser Punkt auch die einzige bedeutsame Veränderung in der Formulierung der späteren Bancroft-Verträge: die nach dem 1. Weltkrieg noch abgeschlos-

10 Vgl. dazu A. N. Makarov, in: Strupp-Schlochauer, Wörterbuch des Völkerrechts, 2. Aufl. (Berlin 1960), Bd. 1, S. 151-3; W. Bazille, Das Recht der Staatsangehörigkeit (Stuttgart 1902), S. 90-107; L. Bendix, Fahnenflucht und Verletzung der Wehrpflicht durch Auswanderung (Leipzig 1906); S. Dzialoszynski, Die Bancroft-Verträge (Breslau 1913); Ch. Munde, The Bancroft Naturalization Treaties with the German States (Würzburg 1868); Italien - U.S. Conciliation Commission, U.S.A. ex rel. Flegenheimer v. Italy, in: AJIL 53 (1959), S. 944-958, bes. $950 \mathrm{ff}$; F. Kapp, der deutsch-amerikanische Vertrag vom 22. Febr. 1868, Preuß. Jahrbücher (1875) Bd. 35, S. 509-534, Bd. 36, S. 189-228. 
senen Verträge dieses Typs sprechen ausdrücklich von der Anerkennung der durch Naturalisation erworbenen neuen Staatsangehörigkeit bei gleichzeitigem Verlust der früheren Staatsangehörigkeit des Auswanderers, lassen also insofern keinen Zweifel mehr bestehen (Bulgarien 1923, Tschechoslowakei 1928, Albanien 1932).

Die Tendenz in der Regelung der Rückwanderungsfolgen ist, wie erwähnt, schon 1868 eindeutig: möglichst nur eine Staatsangehörigkeit, nämlich die des Rückwanderungs- bzw. ursprünglichen Heimatstaates und Verlust der zwischenzeitlich durch Naturalisation erworbenen Staatsangehörigkeit. Aber auch hier gibt es differenzierende Regelungen, die auf unterschiedliche Auffassungen und nationale Staatsangehörigkeits-Vorschriften Rücksicht nehmen. Die häufigste Regelung ist die des Urtyps der Bancroft-Verträge: stillschweigender Verzicht auf die NaturalisationsStaatsangehörigkeit wird bei mindestens zweijährigem Aufenthalt in der ursprünglichen Heimat angenommen (außer den Verträgen von 1868 mit den süddeutschen Staaten z. B. Albanien 1932, Salvador 1908, Brasilien 1908, Dänemark 1872 usw.). Dabei ist offenbar gemeint, daß die individuelle Verzichtserklärung den Verlust der Staatsangehörigkeit automatisch bewirken soll. Das kann sie an sich nur, wenn die (inner-)staatliche Gesetzgebung ihr diese Rechtsmacht verleiht. Diese Wirkung kann mithin nur eintreten, wenn die jeweilige StaatsangehörigkeitsGesetzgebung einen Verlustgrund „Verzicht" ohnehin enthält oder wenn der Bancroft-Vertrag selbst kraft Transformation innerstaatliche Geltung erlangt. Gelegentlich (Baden 1868, Osterreich-Ungarn 1870) wird gesagt, der Rückwanderer sei keineswegs gehalten, seine ursprüngliche Staatsangehörigkeit wieder zu erwerben; tue er es dennoch willentlich, so sei der Verzicht auf die durch Naturalisation erworbene Staatsangehörigkeit (ausdrücklich? Oder soll der Verzicht in dem Wiedererwerb gesehen werden?) möglich.

In anderen Fällen wird aber auch, ähnlich unklar wie in der früher erörterten Frage, lediglich gesagt, der Rückwanderer werde als Staatsangehöriger seines ursprünglichen Heimatstaates betrachtet werden, wenn er dessen Staatsangehörigkeit nach dessen Gesetzen (wieder) erwerbe (Belgien 1868), was zumindest nach heutigen Begriffen nicht mehr als selbstverständlich ist. Die Hauptfrage - nämlich nach dem Schicksal der zwischenzeitlich durch Naturalisation erworbenen Staatsangehörigkeit, nach der etwaigen Doppelstaatsangehörigkeit usw. - bleibt auch hier offen, ähnlich wie in der weiteren Regelung (Schweden - Norwegen 1869, Großbritannien 1870, Dänemark 1872), nach welcher der ursprüngliche Heimatstaat den Rückwanderer auf Antrag nach seinem Recht wieder als Staatsangehörigen aufnehmen $\mathrm{d}$ a $\mathbf{r}$.f. Auch das ist an sich selbstverständlich. Die Regelung verdeutlicht die Konflikte, die es um diese Frage gegeben hat. Und zwar liegt der Ton einmal auf dem Dürfen i m V e rhäl $\mathrm{t} n$ is zu einem anderen, nämlich zum Naturalisierungsstaat, aber zugleich auch auf dem $D$ ü $r f$ e $n$ selbst. Hier geht es darum, daß der ursprüngliche Heimatstaat den Rückwanderer nicht wieder in seinen eigenen Staatsverband aufnehmen $\mathrm{m} u$, was er keineswegs immer freudig zu tun bereit ist.

Die häufig offen bleibende Frage, was mit der durch Naturalisation erworbenen Staatsangehörigkeit bei der Rückwanderung geschieht, wird eindeutig und dem Charakter eines völkerrechtlichen Vertrages am ehesten gemäß dort beantwortet, wo es heißt, der Einwanderungsstaat könne den Rückwanderer, der auf seinen Antrag hin die Staatsangehörigkeit seines ursprünglichen Heimatstaates nach dessen Recht (ohne Aufnahmepflicht) wieder erworben habe, nicht im Hinblick auf die zwischenzeitliche Naturalisation als Staatsangehörigen reklamieren (Großbritan- 
nien 1870, Dänemark 1872, das daneben den stillschweigenden Verzicht kennt). Hier sind Staatsangehörigkeit und Verpflichtungen zwischen den Vertragsparteien am deutlichsten voneinander geschieden. Aber selbst hier kann man nur per argumentum schließen, daß die Naturalisations-Staatsangehörigkeit objektiv, d. h. auch vom Standpunkt des wiederaufnehmenden ursprünglichen Heimatstaates und nicht zuletzt des Betroffenen selbst aus, untergegangen sein soll.

Der dritte in den Bancroft-Verträgen geregelte Gegenstand, der nur aus Anlaß der Staatsangehörigkeits-Bereinigung wegen seiner Wichtigkeit mit behandelt wird, aber nicht selbst die Staatsangehörigkeit berührt, ist die strafrechtliche Verfolgung von Rückkehrern in ihrem ursprünglichen Heimatstaat. Die Mehrzahl der Verträge läßt sie wegen vor der Auswanderung begangener Taten zu. Einige nehmen die Auswanderung selbst als Straftat ausdrücklich aus - ein Hinweis auf den geschlossenen Charakter dieser Gesellschaften (Schweden - Norwegen 1869, Brasilien und Uruguay 1908, mit Einschränkungen Portugal 1908, Costa Rica 1911) oder verbieten umgekehrt eine Strafverfolgung nur wegen der Emigration (Bulgarien 1923, Albanien 1932).

Eine besondere Rolle als möglicher Strafgrund spielte die Umgehung der Wehrpflicht, die ja oft Anlaß für die Auswanderung überhaupt bildete. Insofern wurde eine Strafmöglichkeit gelegentlich, wenn auch mit Einschränkungen, ausdrücklich vorgesehen (Baden 1868, Österreich-Ungarn 1870) oder umgekehrt für grundsätzlich unzulässig und nur in Ausnahmefällen für möglich erklärt (Bulgarien 1923, Tschechoslowakei 1928, Albanien 1932). Nur mit denselben oder ähnlichen Einschränkungen wurde ein Nachdienen gestattet (Baden und Belgien 1868, ÓsterreichUngarn 1870).

IV.

Nach der Staatsangehörigkeit als solcher und unmittelbar ist die Wehrpflicht häufig Gegenstand bilateraler Verträge geworden. Sie gab häufig Anlaß zu Kontroversen zwischen konkurrierenden Heimatstaaten. Das deutet sich schon in den erwähnten Bestimmungen der Bancroft-Verträge an, die die strafrechtlichen Folgen der Verletzung von Wehrdienstvorschriften regeln. Hier geht es indessen darum, $\mathrm{da} ß$ die Tatsache des Militärdienstes sich fast in allen Ländern indirekt auf Erwerb oder Verlust der Staatsangehörigkeit auswirkt.. Die häufigsten Konstellationen sind: Verlust der Staatsangehörigkeit beim Eintritt in fremden Wehrdienst; kein Verlust, solange die eigene Wehrpflicht besteht; erleichterter Erwerb beim Ableisten von Militärdienst im Aufnahmestaat.

Eine erste Gruppe von vertraglichen Vereinbarungen dieser Art ist in Gestalt einfacher Befreiungsklauseln in zahlreichen Handels-, Schiffahrts-, Freundschaftsu. ä. Verträgen seit dem früheren 19. Jahrhundert, aber beschränkt ungefähr auf die Zeit bis zum 1. Weltkrieg, enthalten. Nach dem Muster etwa des Vertrages mit der Schweiz von 1850 (Art. II) versprechen sich die Parteien wechselseitig, Aufenthalter mit der Staatsangehörigkeit der anderen Partei vom Wehrdienst frei zu halten.

Ein weiterer Komplex von Verträgen, die im und seit dem 1. Weltkrieg geschlossen worden sind, knüpft an die Doppelstaatigkeit an. Man kann unter ihnen vollkommen zweiseitige und hinkende Regelungen unterscheiden. Erstere setzen einfach Doppelstaatigkeit voraus, gleich auf welchem Rechtsgrund sie beruht. Wird im Staat des gewöhnlichen Aufenthaltsortes und der engeren Lebensbeziehung - gleich 
welcher der Vertragsstaaten es ist - Wehrdienst geleistet, entfällt die Wehrpflicht im anderen Staat (Schweden 1933, Finnland 1939), oder die Leistung des Wehrdienstes im einen Heimatstaat (ohne die obigen Qualifikationen) gilt sogar als dessen gleichzeitige Erfüllung im anderen Heimat- und Vertragsstaat nach dessen Gesetzen (Frankreich 1948/52, jedoch nur rückwirkend auf die beiden Weltkriege bezogen).

Die hinkenden Verträge berücksichtigen nur einen bestimmten Typ der Doppelstaatigkeit: die durch das Zusammenwirken von ius soli und ius sanguinis begründete. Hat dieser Doppelstaater in seinem Geburtsland seinen gewöhnlichen Wohnsitz, so wird er bei nur zeitweiligem Aufenthalt im anderen Staat - gelegentlich auf zwei Jahre begrenzt in dem Sinne, daß längerer Aufenthalt eine widerlegbare Daueraufenthaltsvermutung begründet und damit zur Wehrpflichtigkeit führt dort nicht wehrpflichtig (Norwegen 1930, Schweiz 1937, Albanien 1932 in einem Vertrag des im übrigen reinen Bancroft-Typs; Litauen 1937 kombiniert diese Vereinbarung mit einer unmittelbaren Regelung der Staatsangehörigkeit - stillschweigender Verzicht - und der Strafbarkeit im Falle der Rückwanderung nach zwischenzeitlicher Naturalisation).

Weniger die Sorge um beiderseitige Staatsangehörige als akute politische Notsituationen lagen einer Reihe von Vereinbarungen $z$ wischen den Vereinigten Staaten und ihren jeweiligen Verbündeten aus Anlaß beider Weltkriege zugrunde. Im Jahre 1918 schlossen die USA mit fünf dieser Staaten förmliche Verträge. Danach unterlagen die Angehörigen des einen Vertragsstaates, die sich im anderen Vertragsstaat aufhielten, dessen Wehrgesetzen, sofern sie nicht binnen bestimmter Fristen in die Streitkräfte des Heimatstaates eintraten oder den Aufenthaltsstaat verließen (Großbritannien und Canada, Griechenland, Frankreich und Italien) ${ }^{\mathbf{1 1}}$.

Im 2. Weltkrieg kamen mit 23 verbündeten Staaten durch Notenaustausch executive agreements ähnlichen Typs zustande: Aufenthalter, die keinen Einbürgerungsantrag gestellt hatten, konnten den Wehrdienst nach ihrer Wahl in den Streitkräften des Aufenthalts- oder des Heimatstaates leisten ${ }^{12}$.

Schließlich finden sich isolierte Wehrpflicht (Staatsangehörigkeits-)Regelungen in allgemeinen Freundschafts- und Handelsverträgen. Von dem Jay-Vertrag mit Großbritannien (1794) abgesehen, der materiell noch Friedensvertrag ist, gibt es je eine Gruppe von Verträgen dieser Art nach dem 1. und dem 2. Weltkrieg. Die erste Gruppe erkennt wörtlich übereinstimmend nach dem Vorbild des Vertrages mit Deutschland 1923 im Falle des Krieges eines Vertragsstaates mit einem Drittstaat an, daß die Angehörigen des anderen Vertragsstaates, die sich in dem kriegführenden Vertragsstaat dauernd und mit erklärter Einbürgerungsabsicht aufhalten, der Wehrpflicht des letztgenannten Staates unterliegen, sofern sie nicht binnen 60 Tagen nach Kriegserklärung abreisen (Estland und Ungarn 1925, Salvador 1926, Lettland, Ósterreich und Norwegen 1928, Liberien 1938).

Die untereinander ebenfalls praktisch identischen Klauseln in den Handelsverträgen nach 1945 sind komplizierter. Zunächst sichert jeder Partner dem anderen die Befreiung seiner Staatsangehörigen vom Wehrdienst zu. Diese Befreiung entfällt d. h. der Wehrdienst ist in den Streitkräften des anderen Vertragsstaates zu leisten - außer im Falle der Doppelstaatigkeit ${ }^{13}$ beim gemeinsamen Kampf beider Vertragsstaaten gegen Dritte oder - ein Zeichen für frühen Nachkriegs-Optimis-

\footnotetext{
11 Dazu L. H. Woolsey, The military service conventions between the United States and associated countries, AJIL 12 (1918), S. 824-828.

12 Zusammenstellung dieser Ablkommen bei Whiteman, S. 569-571. Näheres unten im Dokumententeil.

13 So z. B. Protokoll Ziff. 3 zum Vertrag mit Irland 1950, UNTS Bd. 206, S. 298, 300.
} 
mus - bei gemeinsamen Maßnahmen zur Friedenssicherung usw. im Sinne der UN-Satzung. Aber auch hier wird wieder eine Befreiung vom Wehrdienst in den fremden Streitkräften vereinbart, wenn der Aufenthalter, der keinen Einbürgerungsantrag gestellt hat, binnen angemessener Frist in den Streitkräften seines Heimatstaates zu dienen vorzieht (China 1946, Italien 1948, Irland 1950. Die stattliche Reihe der nach diesem Zeitpunkt noch geschlossenen Freundschaft-, Niederlassungs-, Handels-, Schiffahrts- usw. Verträge enthält keine die Staatsangehörigkeit unmittelbar oder mittelbar - über die Wehrpflicht - behandelnden Klauseln mehr).

Diese Wehrpflichtabkommen im weiteren Sinne haben unter den folgenden dargestellten Aspekten des amerikanischen Staatsangehörigkeits-Rechts - vom Recht der zahlreichen Vertragspartner wird hier abgesehen - Auswirkungen auf die Staatsangehörigkeit selbst.

Zunächst geht es um Amerikaner, die in fremden Staaten Wehrdienst leisten. In den Doppelstaaterverträgen, in den Wehrpflichtabkommen beider Weltkriege und schließlich in den nach 1918 entstandenen Wehrpflichtklauseln der Freundschafts- und Handelsverträge erkennt jeweils ein Vertragspartner qua Heimatstaat an, daß sein eigener Staatsangehöriger, der sich im anderen Vertragsstaat aufhält, unter bestimmten jeweils spezifizierten Bedingungen der Wehrpflicht des Aufenthaltsstaates unterworfen ist. Mitunter wird schon im Wortlaut klargestellt (so im Doppelstaaterabkommen mit Frankreich), daß der Betroffene nicht oder nur noch unter besonderen Umständen im Heimatstaat Wehrdienst leisten muß, so wenn er ein entsprechendes Wahlrecht ausübt wie in den Handelsverträgen nach 1945. Ja nach dem Doppelstaaterabkommen mit Frankreich gilt der in einem Staat geleistete Wehrdienst ausdrücklich zugleich als Erfüllung der Wehrpflicht des anderen. Diese Regelungen betreffen aber zunächst nur die Wehrdienstfrage im Verhältnis der Vertragsstaaten zueinander. Nach amerikanischem Staatsangehörigkeits-Recht führt nun aber der Eintritt in fremde Streitkräfte ohne ausdrückliche Genehmigung durch die Secretaries of State und Defense normalerweise zum Verlust der amerikanischen Staatsangehörigkeit ${ }^{14}$. Der Einfluß der erwähnten Wehrdienstverträge auf diese Bestimmung wird meist nicht explizit gemacht. Nur die Wehrdienstverträge von 1918 schließen ausdrücklich diese Verlustwirkung aus. Dieser zunächst zwischenstaatlichen Vereinbarung kommt im Transformationswege dann allerdings zugleich unmittelbare interne staatsangehörigkeitsrechtliche Wirkung zu. Dasselbe muß aber auch für die anderen Wehrdienstverträge im weiteren Sinne gelten. Wenn der Sinn der Verlustbestimmungen darin liegt, daß die Anerkennung einer Wehrpflicht in einem fremden Staate unvereinbar mit den Pflichten des amerikanischen Bürgers den Vereinigten Staaten gegenüber ist ${ }^{15}$, dann bedeutet eben der Wehrdienstvertrag, daß die Vereinigten Staaten im geregelten Umfange diese Unvereinbarkeit verneinen und mithin die amerikanische Staatsangehörigkeit nicht verlorengeht, wenn ein Amerikaner im Rahmen des Wehrdienstvertrages in einem fremden Staate (dem Vertragsstaat) Wehrdienst leistet. Diese Argumentation ist entscheidend. Denn wenn man nur auf die Zeitfolge der Normen abstellt, würde jede diesen Verlustgrund wiederum enthaltende Neufassung des Staatsangehörigkeitsgesetzes nach amerikanischer Auffassung dem früheren Vertrage

14 Immigration and Nationality Act 1952, \349 (a) (3), Stat. Bd. 66, S. $267=$ U. S. C. Title 8, \1481 (a) (3). 15 U. S. ex rel. De Cicco v. Longo, D. C. Com. 1942, F. Supp. Bd. 46, S. 170. 
in seiner innerstaatlichen Wirkung ohne weiteres derogieren (der Vertrag hat keine höherrangige Geltung). - Entsprechendes würde für die Vertragspartner der Vereinigten Staaten gelten.

Außer den bisher besprochenen Vertragsnormen für eigene Staatsangehörige, die fremden Wehrdienst leisten, stipulieren wie dargelegt zahlreiche Vertragsklauseln die gegenseitige Befreiung vom Wehrdienst. Hier verspricht ein Staat dem anderen, die Staatsangehörigen des Vertragspartners, die sich im Gebiet des ersteren aufhalten, nicht zum Wehrdienst heranzuziehen. Hier geht es also um die Rechtslage fremder Staatsangehöriger, die in den USA vom Wehrdienst befreit sind.

Diese Regelung kann sich naturgemäß nicht auf den Verlust einer noch nicht existenten, aber doch auf die Möglichkeiten des künftigen Erwerbs der Staatsangehörigkeit der Vereinigten Staaten auswirken. Zunächst haben die Vereinigten Staaten unter Verletzung dieser völkerrechtlichen Verträge in beiden Weltkriegen auch vertraglich befreite Ausländer zum Wehrdienst herangezogen ${ }^{16}$, da die Wehrgesetze grundsätzlich auf alle männlichen Personen anzuwenden sind ${ }^{17}$ und sie, wie erwähnt, soweit später erlassen, jedenfalls innerstaatlich dem Vertrag vorgehen ${ }^{18}$. Abgesehen von der hier nicht $\mathrm{zu}$ verfolgenden Gefährdung der Rechtsstellung des Ausländers in seinem Heimatstaat verbesserte diese amerikanische Praxis seine Position im Hinblick auf einen beabsichtigten Erwerb der amerikanischen Staatsangehörigkeit, denn die Einbürgerung wird auch in den Vereinigten Staaten erleichtert, wenn der Antragsteller in den amerikanischen Streitkräften dient ${ }^{19}$. Gegenvorstellungen von Partnerstaaten und des Department of State wegen dieser Vertragsverletzung führten schließlich dazu, daß die amerikanische Gesetzgebung die Befreiungsklauseln berücksichtigt, aber nur in der Weise, daß jemand, der sich auf sie beruft, zwar nicht in den amerikanischen Streitkräften zu dienen braucht, dafür aber vom Erwerb der amerikanischen Staatsangehörigkeit schlechthin ausgeschlossen wird. Die Befreiungsklausel in Verbindung mit dem inneramerikanischen Recht stellt also den vertraglich begünstigten Ausländer vor die Alternative, entweder sich auf die vertraglich vorgesehene Befreiung zu berufen, dann aber nie amerikanischer Bürger werden zu können, oder auf sie zu verzichten, d. h. in den USA Soldat zu werden, und dann die amerikanische Staatsangehörigkeit (sogar beschleunigt) erwerben zu können ${ }^{20}$. Lediglich der Vertrag mit China 1946 enthält neben der Befreiungsklausel einen warnenden Hinweis auf die Möglichkeit einer derartigen Regelung ${ }^{21}$. Die Vergünstigung der Befreiung wird vom amerikanischen Gesetz zwingend an den erwähnten Nachteil gekoppelt ${ }^{22}$.

Schließlich ist in diesem Zusammenhange noch das Wanderarbeiterabkommen mit Mexiko 1951 (das z. Z. letzte in einer Reihe mehrerer ähnlicher Abkommen) zu erwähnen. Es enthält nicht nur die Befreiungsklausel, sondern schließt die betroffenen Mexikaner darüber hinaus positiv von jedem amerikanischen Wehrdienst aus. Es eröffnet also nicht wie sonst durch die Befreiungsklausel in Verbindung

$16 \mathrm{Vgl}$. Whiteman, S. $549 \mathrm{ff}$.

17 Vgl. zuletzt den Military Selective Service Act 1967, \ 1 (2) mit Vorläufern, Stat. Bd. 81, S. 100, U. S. C. Title 50 App. 454 (a)

18 Hierzu Moser v. U. S., U. S. Reports Bd. 341 (1951) S. 41, 45.

19 Immigration and Nationality Act 1952, SS $328 \mathrm{ff}$., Stat. Bd. 66, S. 249, U. S. C. Title 8, SS $1439 \mathrm{ff}$.

20 Vgl. zuletzt Immigration and Nationality Act 1952 \$ 315, Stat. Bd. 66, S. 242, U. S. C. Title 8, \$ 1426.

21 Versteckt ebenso die Minutes of Interpretation zum Vertrag mit Irland 1950 zu Art. 3 Abs. 1, in: UNTS Bd. 206, S. $304=$ UST Bd. 1, S. 808 .

22 Durch eine executive order vom 24. 11. 1970 hat der amerikanische Präsident im Hinblick auf die Interessen amerikanischer Firmen mit Auslandsniederlassungen eine echte Möglichkeit der Befreiung vom Wehrdienst verfügt für solche Ausländer, die von amerikanischen Gesellschaften im Ausland in "executive, managerial or special posts" beschäftigt werden und im Zusammenhang mit ihrer Tätigkeit in die USA kommen. Die Folge: sie können gleichwohl eingebürgert werden; die Ausschluß-Alternative gilt für sie nicht. Nach Internat. Herald Tribune vom 26. 11. 1970. 
mit der internen Gesetzgebung die Wahlmöglichkeit zwischen Befreiung und Wehrdienst mit erleichterter Einwanderung, sondern verschließt ausdrücklich diese letzte Alternative. Man wollte dem besonderen Status dieser zahlreichen Mexikaner als Wanderarbeiter - soziologisch ein ganz anderer Typus als der Daueraufenthalter mit Einbürgerungsabsicht - Rechnung tragen und zugleich verhindern, daß sich hier eine Einwanderungspforte eigener Art für eine größere Zahl möglicher Interessenten außerhalb des ordentlichen Einwanderungssystems auftue.

\section{V.}

Eine vorwiegend historisch interessante, später jedoch nicht wieder in Erscheinung tretende Regelung mit indirekter Auswirkung auf die Staatsangehörigkeit findet sich übereinstimmend in den Verträgen mit Nicaragua 1867 (Art. 9 Abs. 1) und Salvador 1870 (Art. 29 Abs. 1). Sie setzte die bis vor kurzem fast universal anzutreffende Norm außer Kraft, nach welcher die Eheschließung mit einem Ausländer den Staatsangehörigkeitswechsel der Frau zur Folge hat. Die erwähnten Vertragsnormen bestimmten ausdrücklich, daß diese Wirkung nicht eintreten sollte. Man darf vermuten, daß das Interesse der auch damals im Verhältnis zu den Vertragspartnern schon übermächtigen USA an der Erhaltung der Staatsangehörigkeit der Amerikanerin dieser Vereinbarung zugrunde lag. Folgewirkungen hat sie nicht gezeitigt.

Als mehr prozedurale denn substantielle Besonderheit sei abschließend ein Versuch erwähnt, für die Behandlung der zwischen zwei Staaten auftauchenden Staatsangehörigkeits-Streitfragen eine besondere gemeinsame Instanz zu schaffen. In der Regel entscheiden nationale Stellen (Verwaltung, Gerichte) diese Probleme, seltener internationale Gerichte (die Inzident-Entscheidung Nottebohm ist eine Ausnahme). Gelegentlich sind zwischenstaatliche gemischte Schiedsgerichte, Schiedskommissionen usw. berufen, im Zusammenhang mit Entschädigungsfragen inzidenter über die Staatsangehörigkeit zu erkennen ${ }^{23}$. Im Jahre 1947 machten nun die Vereinigten Staaten und Polen den interessanten Versuch, eine gemeinsame Staatsangehörigkeits-Kommission einzurichten, die sich ausschließlich den zwischen beiden Ländern etwa streitig werdenden Staatsangehörigkeits-Fällen widmen sollte, soweit ersichtlich ein Novum der zwischenstaatlichen Beziehungen. Allerdings hatte die Kommission keine selbständige Entscheidungsgewalt. Sie sollte nach Sachprüfung lediglich Empfehlungen für die beiden Regierungen formulieren. - Bedeutsam ist an ihr ohnehin nur die Idee. Sie wurde nämlich ein Opfer des sich entfaltenden Kalten Krieges. Nachdem sie gar nicht praktisch hatte tätig werden können, wurde sie 1948 wieder aufgelöst.

Ähnlich dem Verfahren mehr als der Sachregelung verhaftet ist eine amerikanischjugoslawische Vereinbarung von 1950. Sie betraf Fälle möglicher Doppelstaatigkeit, ließ dieses Problem selbst jedoch offen und beschränkte sich auf eine Art Garantie freier Ausreise für amerikanische Staatsangehörige jugoslawischer Abstammung, die mit jugoslawischem Visum nach Jugoslawien eingereist waren, auch wenn dieser Staat sie als seine eigenen Angehörigen beanspruchte.

$23 \mathrm{Vgl}$. die oben, Anm. 10, zitierte Entscheidung. 


\section{DOKUMENTATION}

\section{Inhaltliche Übersicht}

Die das Staatsangehörigkeits-Recht direkt betreffenden oder für dieses indirekt erheblichen Verträge der USA umfassen folgende fünf Gruppen: Einbürgerungsverträge, Wehrdienstverträge, Verträge über Gebietsabtretung, Sonderverträge sowie fünftens multilaterale Verträge.

1. Die Gruppe der Einbürgerungsverträge umfaßt folgende Verträge mit: Albanien, Belgien, Brasilien (1951 gekündigt), Bulgarien, Costarica, Dänemark, Deutschland (Norddeutscher Bund und vier süddeutsche Staaten, außer Kraft 1917), Ecuador (1892 gekündigt), Großbritannien (1953 gekündigt), Haiti, Honduras, Mexico (1882 gekündigt), Nicaragua, Österreich-Ungarn (außer Kraft 1917), Peru, Portugal, Salvador, Schweden - Norwegen, Tschechoslowakei, Türkei (Inkrafttreten verhindert), Uruguay. Von diesen 25 Verträgen sind sechs durch den ersten Weltkrieg außer Kraft getreten, einer konnte nicht in Kraft treten und vier wurden gekündigt. Es sind also noch 14 dieser sog. Bancroft-Verträge in Kraft. Alle vier Kündigungen gingen nicht von den USA aus, sondern von ihren Partnern.

2. Die Gruppe der Wehrdienstverträge umfaßt vier Untertypen:

a) 8 Doppelstaaterabkommen mit: Finnland, Frankreich (3 Verträge), Norwegen, Schweden, Schweiz, Litauen; ferner Bestimmung im Einbürgerungsvertrag mit Albanien (s. o.).

b) 5 Wehrdienstverträge mit den Alliierten von 1918: Frankreich, Griechenland, Großbritannien (mit Canada), Italien. Ähnliche Verträge aus dem Zweiten Weltkrieg 1942-1945 mit 23 Alliierten: Australien, Belgien, Brasilien, Canada, Chile, China, Columbien, Cuba, Ecuador, Griechenland, Großbritannien, Indien, Jugoslawien, Mexico, Neuseeland, Niederlande, Norwegen, Peru, Polen, Salvador, Südafrika, Tschechoslowakei, Venezuela sowie ein zweiter Vertrag mit Canada.

c) 12 Handelsverträge mit Klausel: China, Deutschland (außer Kraft 1941), Großbritannien (s. aber auch Gruppe 3), Irland, Italien, Liberia, Norwegen, Osterreich, Salvador (außer Kraft 1958), Estland, Lettland, Ungarn.

d) 23 Verträge (meist ältere Handelsverträge) mit Klausel über Befreiung vom Wehrdienst mit: Argentinien, Costarica, Dominikanische Republik, Haiti, Hawaii, Honduras, Jugoslawien (Serbien), Italien (2mal), Japan (2mal), Kongo, Mexico, Nicaragua, Paraguay, Salvador, Schweiz, Spanien, Südafrika, Thailand (2mal), Tonga, Venezuela.

3. Staatsangehörigkeitsvorschriften aus Anlaß von Gebietsabtretungen finden sich im Verhältnis zu folgenden Staaten: Dänemark (Westindien), Frankreich (Louisiana), Mexico (Texas, Arizona), Sowjetunion (Alaska), Spanien (Florida und Kolonien von 1898).

4. Sonderverträge betrafen Staatsangehörigkeits-Kommission (Polen, außer Kraft 1948), Visumfragen (Jugoslawien), Verweisung auf Art. 278 Versailler Vertrag (Deutschland), Staatsangehörigkeit der Südsee-Inseln (Vereinte Nationen).

5. Multilaterale Verträge: Interamerikanische Abkommen über Status Eingebürgerter (13. 8. 1906) und über Staatsangehörigkeit der Ehefrau (26. 12. 1933); Haager Protokoll über Wehrdienst (12. 4. 1930); Marokko-Vertrag (3. 7. 1880). 


\section{Alphabetische Übersicht}

Die mit einem Stern versehenen Verträge sind außer Kraft getreten, die mit zwei Sternen sind nie ratifiziert worden.

$$
\begin{aligned}
& \mathrm{A}=\text { Abtretungs-Vertrag (Gebietswechsel) } \\
& \mathrm{B}=\text { Befreiungs-Klausel (Wehrpflicht) } \\
& \mathrm{D}=\text { Doppelstaater-Vertrag (Wehrpflicht) } \\
& \mathrm{E}=\text { Einbürgerungs-Vertrag } \\
& \mathrm{H}=\text { Handels-Vertrag mit Klausel } \\
& \mathrm{S}=\text { Sonder-Vertrag } \\
& \mathrm{W}=\text { Wehrdienst-Vertrag beider Weltkriege }
\end{aligned}
$$

\begin{tabular}{|c|c|c|c|}
\hline & Albanien & 5. 4.1932 & $\mathrm{E}$ \\
\hline & Argentinien & 27. 7.1853 & B \\
\hline$*$ & Australien & 31. 3.1942 & W \\
\hline & Belgien & 16. 11.1868 & $\mathrm{E}$ \\
\hline$*$ & Belgien & 31. 3. 1942 & W \\
\hline * & Brasilien & 27. 4. 1908 & $\mathrm{E}$ \\
\hline * & Brasilien & 23. 1.1943 & W \\
\hline & Bulgarien & 23. 11.1923 & E \\
\hline * & Canada & 3. 6.1918 & W \\
\hline * & Canada & 18. 3.1942 & W \\
\hline$*$ & Canada & 30. 3.1942 & W \\
\hline * & Chile & 7. 6.1945 & W \\
\hline$*$ & China & 6.11 .1943 & W \\
\hline & China & 4.11 .1946 & $\mathrm{H}$ \\
\hline * & Columbien & 27. 1. 1944 & W \\
\hline & Costarica & 10. 7. 1851 & B \\
\hline & Costarica & 10. 6. 1911 & $\mathrm{E}$ \\
\hline * & Cuba & 6. 11.1942 & W \\
\hline & Dänemark & 20. 7.1872 & E \\
\hline & Dänemark & 4. 8.1916 & A \\
\hline * & Deutschland & 22. 2. 1868 & E \\
\hline & Deutschland & 25. 8.1921 & $\mathrm{~S}$ \\
\hline * & Deutschland & 8. 12.1923 & $\mathrm{H}$ \\
\hline * & Baden & 19. 7.1868 & $\mathrm{E}$ \\
\hline * & Bayern & 25. 5.1868 & $\mathrm{E}$ \\
\hline * & Hessen & 1. 8.1868 & $\mathbf{E}$ \\
\hline * & Württemberg & 27. 7.1868 & $\mathrm{E}$ \\
\hline * & Dominikanische Republik & 8. 2. 1867 & B \\
\hline * & Ecuador & 6. 5. 1872 & $\mathrm{E}$ \\
\hline * & $\begin{array}{l}\text { Ecuador } \\
\text { Estland (s. Sow jetunion) }\end{array}$ & 2. 4.1945 & W \\
\hline & Finnland & 27. 1.1939 & $\mathrm{D}$ \\
\hline & Frankreich & 30. 4.1803 & $\mathrm{~A}$ \\
\hline * & Frankreich & 3. 9.1918 & W \\
\hline$*$ & Frankreich & 25. 2. 1948 & $\mathrm{D}$ \\
\hline & Frankreich & 22. 12. 1948 & D \\
\hline & Frankreich & 31. 12. 1952 & $\mathrm{D}$ \\
\hline * & Griechenland & 30. 8.1918 & W \\
\hline * & Griechenland & 31. 3. 1942 & W \\
\hline * & Großbritannien & 19. 11.1794 & $\mathrm{H}(\mathrm{A})$ \\
\hline * & Großbritannien & 13. 5.1870 & $\mathrm{E}$ \\
\hline & Großbritannien & 23. 2. 1871 & $\mathrm{E}$ \\
\hline * & Großbritannien & 3. 6.1918 & W \\
\hline 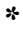 & Großbritannien & 30. 3.1942 & W \\
\hline * & Haiti & 3. 11.1864 & B \\
\hline & Haiti & 22. 3. 1902 & $\mathrm{E}$ \\
\hline & Haiti & 28. 2. 1903 & E \\
\hline
\end{tabular}




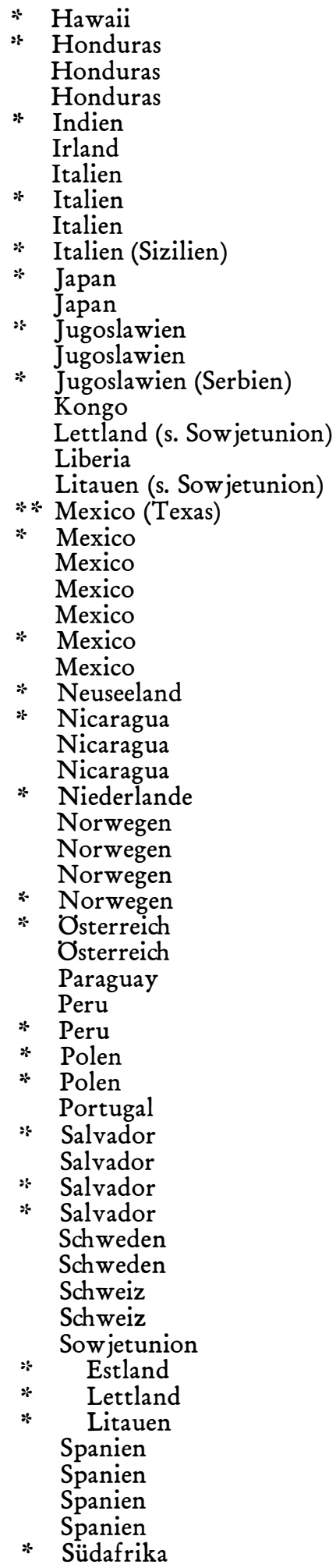

20.12.1849 B

4. 7. 1864 B

23. 6. 1908 E

7. $12.1927 \mathrm{H}$

30. 3. 1942 W

21. $1.1950 \mathrm{H}$

26. 2. 1871 B

24. 8. 1918 W

2. 2. $1948 \mathrm{H}$

1. $10.1855 \quad \mathrm{~B}$

22. 11. $1894 \quad B$

21. 2.1911 B

31. 3.1942 W

23. 3. $1950 \mathrm{~S}$

14. 10. 1881 B

24. 1. 1891 B

8. 8. $1938 \mathrm{H}$

12. 4. $1844 \mathrm{~A}$

5. 4. 1831 B

2. 2. $1848 \mathrm{~A}$

30. 12. $1853 \mathrm{~A}$

10. 7. $1868 \mathrm{E}$

22. 1. 1943 W

11. 8. 1951 B

31. 3.1942 W

21. 6. 1867 B

7. 12. $1908 \mathrm{E}$

17. 6. $1911 \mathrm{E}$

31. 3.1942 W

26. 5. $1869 \mathrm{E}$

5. 6. $1928 \mathrm{H}$

1. $11.1930 \mathrm{D}$

31. 3. $1942 \mathrm{~W}$

20. $9.1870 \mathrm{E}$

19. 6. $1928 \mathrm{H}$

4. 2. 1859 B

15.10.1907 E

23. 5. 1945 W

30. 3.1942 W

16. 2. $1947 \mathrm{~S}$

7. 5. $1908 \mathrm{E}$

6. $12.1870 \quad \mathrm{~B}$

14. 3. $1908 \mathrm{E}$

22. 2. $1926 \mathrm{H}$

3. $4.1943 \mathrm{~W}$

26. 5. 1869 E

31. 1.1933 D

25.11.1850 B

11. 11. 1937 D

30. 3. $1867 \quad \mathrm{~A}$

23. 12. $1925 \quad \mathrm{H}$

20. 4. $1928 \mathrm{H}$

18. 10.1937 D

22. 2. 1819 A

10.12.1898 A

29. 2. 1900 A

3. $7.1902 \mathrm{~B}$

31. 3. 1942 W 
* Südafrika (Oranjefreistaat)

22. 12. $1871 \quad \mathrm{~B}$

* Thailand 16.12.1920 B Thailand 13.11.1937 B

Tonga (StAB außer Kraft) 2. 10.1886 B

Tschechoslowakei 16. 7.1928 E

* Tschechoslowakei 3. 4. 1942 W

* Türkei 11. 8.1874 E

* Ungarn 20. 9.1870 E

* Ungarn 24. 6.1925 H

Uruguay 10. 8.1908 E

* Venezuela 27. 8.1860 B

* Venezuela 10. 5.1945 W

Vereinte Nationen $\quad$ 2. 4. 1947 S

Anhang (Multilaterale)

Völkerbund

Amerikanische Staaten

Amerikanische Staaten

12. 4.1930

13. 8.1906

26. 12.1933

Vierzehnmächte (Marokko)

3. 7.1880 


\section{Chronologische Übersicht}

Die Daten in Klammern unter dem Datum des Vertragsschlusses bezeichnen das Inkrafttreten. Unter „Prokl.“ ist die Proklamation des amerikanischen Präsidenten $\mathrm{zu}$ verstehen, mit welcher das Inkrafttreten festgestellt wird.

19. 11.1794 Freundschafts-, Handels- und Schiffahrtsvertrag mit Großbritan(28. 10.1795) nien (sog. Jay-Vertrag)

Dagegen hatte der Friedensvertrag v. 3. 9. 1783 keine StAB enthalten. England nahm StA-Erwerb aber ab 1783 an, die USA dagegen schon ab der Unabhängigkeitserklärung v. 1776 ( s. Inglis v. Sailor's Snug Harbour)

StAB: Art. 2

Prokl.: 29. 2. 1796

Amtl. Text: Statutes, Bd. 8, S. 116

TS 105

Engl. Text: State Papers Bd. 1, S. 784

Malloy, S. 590

Miller, Bd. 2, S. 245

Engl. u. franz. Text: Martens, R., Bd. 6, S. 336

Dt. Úbers. v. Art. 2: Rabl, S. 133

Fälle: Crane v. Reeder

30. 4. 1803 Vertrag mit Frankreich über die Abtretung von Louisiana

(21. 10.1803) StAB: Art. 3

Prokl.: 21.10. 1803

Amtl. Text: Statutes, Bd. 8, S. 200

TS 86

Engl. u. franz. Text: Martens, R., Bd. 10 (Suppl. Bd. 3), S. 464

Engl. Text: Malloy, S. 508

Hyatt, S. 1227

Miller, Bd. 2, S. 498

Dt. Übers. v. Art. 3: Rabl, S. 131

Fälle: Aubry v. U. S.; de Baca v. U. S.; Desbois v. U. S.;

Foucher v. U.S.; Scott and U.S. v. Laverty;

State v. Primrose

22. 2. 1819 Vertrag mit Spanien betr. u. a. die Abtretung von Florida

(22. 2. 1821) StAB: Art. 6

Prokl.: 22. 2. 1821

Amtl. Text: Statutes, Bd. 8, S. 252

TS 327

Engl. u. span. Text: State Papers Bd. 8, S. 524

Martens, N.R. Bd. 5, S. 328

Engl. Text: Malloy, S. 1651

Hyatt, S. 1243

Miller, Bd. 3, S. 3

Dt. Übers. v. Art. 6: Rabl S. 131

Fälle: American Ins. Co. v. Canter; Boyd v. Thayer;

Tannis v. Doe ex dem. St. Cyre

5. 4. 1831 Handels-, Freundschafts- und Schiffahrtsvertrag mit Mexiko

(5. 4. 1832) Suspendiert durch Krieg v. 1846/7; wieder in Kraft durch Art. 17 des Vertrages v. 2. 2. 1848 (s. u.); außer Kraft durch Kündigung Mexikos am 30.11.1881

StA/WdB: Art. 9

Prokl.: 5. 4. 1832

Amtl. Text: Statutes Bd. 8, S. 410

TS 203

Engl. Text: Malloy, S. 1084

State Papers, Bd. 19, S. 209 
Martens, N.R., Bd. 10, S. 322

Miller, Bd. 3, S. 599

Fälle: Atocka v. U.S.

12. 4. 1844 Vertrag mit der Republik Texas über deren Beitritt zu den USA StAB: Art. 2

Vom amerikanischen Senat nicht ratifiziert, daher nie in Kraft. Texas, das 1836 unabhängig geworden war, wurde 1845 ohne Vertrag von den USA annektiert. Durch Prokl. v. Gen. Kearny v. 22. 8. 1846 über die Annexion New Mexicos wurde ein automat. StA-Wechsel festgestellt (Martens, N. R. G., Bd. 9, S. 320)

Engl. Text: State Papers Bd. 33, S. 262

Martens, N.R.G., Bd. 6, S. 378

Miller, Bd. 4, S. 697

2. 2. 1848 Vertrag von Guadalupe Hidalgo mit Mexiko über Frieden,

(30. 5. 1848) Freundschaft, Grenzen und Ausgleich

StAB: Art. 8-9 (betr. Texas)

Prokl.: 4. 7. 1848

Amtl. Text: Statutes Bd. 9, S. 922

TS 207

Engl. Text: State Papers Bd. 37, S. 567

Hyatt, S. 1235

Engl. und span. Text: Martens, N.R.G., Bd. 14, S. 7

Miller, Bd. 5, S. 207

Malloy, S. 1107

Dt. Übers.: Martens, N.R.G., Bd. 11, S. 387

Rabl, S. 132 (nur Art. 8)

Fälle: bei Dyne, S. 284-291, 329

20. 12. 1849 Freundschafts-, Handels- und Schiffahrtsvertrag mit Hawaii

(24. 8. 1850) Außer Kraft mit der Annexion durch USA am 7. 7.1898

StAB/WdB: Art. 8

Prokl.: 9.11. 1850

Amtl. Text: Statutes Bd. 9, S. 977

TS 160

Engl. Text: Malloy, S. 908

Martens, N.R.G., Bd. 14, S. 680

Miller, Bd. 5, S. 591

25. 11.1850

(8. 11. 1855)

Vertrag über Freundschaft, Handel und Auslieferung

mit der Schweiz

StAB/WdB: Art. 2 Abs. 1

Prokl.: 9. 11. 1855

Amtl. Text: Statutes Bd. 11, S. 587

TS 353

Englischer Text: Malloy, S. 1763

State Papers Bd. 38, S. 285

Franz. Text: Martens, N. R. G., Bd. 16 I, S. 25

Fälle: Haver v. Yaker

10.7.1851 Freundschafts-, Handels- und Schiffahrtsvertrag

(26. 5. 1852) mit Costarica

StA/WdB: Art. 9

Prokl.: 26. 5. 1852

Amtl. Text: Statutes Bd. 10, S. 916

TS 62

Engl. Text: Malloy, S. 341

State Papers Bd. 40, S. 1090

Martens, N.R.G., Bd. 16 I, S. 113

Miller, Bd. 5, S. 985 
27. 7.1853

(20.12.1854)

Freundschafts-, Handels- und Schiffahrtsvertrag

mit Argentinien

StA/WdB: Art. 10

Prokl.: 9. 4. 1855

Amtl. Text: Statutes Bd. 10, S. 1005

TS 4

Engl. Text: Malloy, S. 20

State Papers Bd. 42, S. 720

Miller, Bd. 6, S. 269

30. 12. 1853

(30.6. 1854)

1. 10.1855

(7. 11. 1856)

4. 2. 1859

(7. 3. 1860)

27. 8.1860

(9. 8. 1861)

4.7.1864 Freundschafts-, Handels- und Schiffahrtsvertrag mit Honduras

(5. 5. 1865) Außer Kraft mit Inkrafttreten des neuen Vertrages v. 7.12.1927 (s. u.) am 19. 7. 1928

StA/WdB: Art. 9

Prokl.: 30. 5. 1865

Amtl. Text: Statutes, Bd. 13, S. 6 .. 9

bzw. Bd. 18 II, S. 426

TS 172

Engl. Text: Malloy, S. 952

State Papers Bd. 54, S. 1134 
3. 11. 1864 Freundschafts-, Handels-, Schiffahrts- und

(22. 5. 1865) Auslieferungsvertrag mit Haiti

Außer Kraft am 7. 5. 1905 durch Kündigung seitens Haitis

StA/WdB: Art. 5

Prokl.: 6. 7. 1865

Amtl. Text: Statutes Bd. 13, S. 711

TS 164

Engl. Text: Malloy, S. 921

State Papers Bd. 54, S. 1141

8. 2. 1867 Konvention über Freundschaft, Handel, Schiffahrt und Auslieferung

(5. 10. 1867) mit der Dominikanischen Republik

Außer Kraft am 13.1.1898 durch Kündigung seitens der Dominikanischen Republik

StA/WdB: Art. 2

Prokl.: 24. 10. 1867

Amtl. Text: Statutes Bd. 15, S. 473

TS 73

Engl. Text: Malloy, S. 403

State Papers Bd. 59, S. 245

30. 3. 1867 Vertrag mit Rußland über die Abtretung Alaskas

(20.6. 1867) StAB: Art. 3

Prokl.: 20. 6. 1867

Amtl. Text: Statutes Bd. 15, S. 542

TS 301

Engl. Text: Martens, N.R.G. ${ }^{2}$, Bd. 1, S. 39

State Papers Bd. 57, S. 452

Malloy, S. 1521

Flournoy, S. 578

Hyatt, S. 1241

Dt. Úbers. v. Art. 3: Rabl, S. 132

Fälle: Rasmussen v. U.S. (1905); Levy (in: Moore III, 2514)

21. 6.1867 Freundschafts-, Handels- und Schiffahrtsvertrag mit Nicaragua

(20.6.1868) außer Kraft am 24.10.1902 durch Kündigung Nicaraguas

StA/WdB: Art. 9 Abs. 2

Prokl.: 13. 8. 1868

Amtl. Text: Statutes Bd. 15, S. 549

TS 257

Engl. Text: Malloy, S. 1279

State Papers, Bd. 58, S. 1162

22. 2. 1868 Einbürgerungsvertrag mit dem Norddeutschen Bund

(9. 5. 1868) Dieser erste der sogenannten Bancroft-Verträge galt bis zum Kriegseintritt der USA von 1917. Deutschland wandte ihn auf ElsaßLothringen nicht an.

Prokl.: 27. 5. 1868

Amtl. Text: BGBl. 1868, S. 228

Statutes Bd. 15, S. 615

TS 261

Dt. Text: Sieber Bd. 2, S. 343

Cahn, 1. Aufl., S. 174

Engl. Text: State Papers, Bd. 58, S. 1170

Malloy, S. 1298

Martens, N.R.G., Bd. 19, S. 78

Dyne, S. 466

Franz. Text: Zeballos, Bd. 4, S. 388

Anwendung: Hack worth, S. 384-386 
26. 5.1868

(18. 9. 1868)

10.7 .1868

(1. 2. 1869)

19.7. 1868

(7. 12.1869)

27.7. 1868

(17. 8. 1869)

1. 8. 1868

(23. 7. 1869)
Einbürgerungsvertrag mit dem Königreich Bayern

mit Protokoil vom gleichen Tage (Cahn, 1. Aufl., S. 402)

Prokl.: 8. 10. 1868

Amtl. Text: RegBl. 1868, S. 2153

Statutes Bd. 15, S. 661

TS 18

Dt. Text: Sieber, Bd. 2, S. 345

Engl. Text: State Papers Bd. 58, S. 988

Malloy, S. 60

Dyne, S. 447 (Protokoll S. 449)

\section{Einbürgerungsvertrag mit Mexico}

Außer Kraft: 11. 2. 1882 (State Papers Bd. 72, S. 1193)

durch Kündigung Mexicos

Prokl.: 1. 2. 1869

Amtl. Text: Statutes Bd. 15, S. 687

TS 213

Engl. Text: State Papers Bd. 59, S. 263

Malloy, S. 1132

Franz. Úbers.: Zeballos, Bd. 2, S. 46 (Art. 1-5);

Bd. 4, S. 633 (Art. 1 und 4)

Fälle: in re Rodriguez

\section{Einbürgerungsvertrag mit Baden}

Prokl.: 10. 1. 1870

Amtl. Text: VOBl. 1869, S. 579

Statutes Bd. 16, S. 731

TS 15

Dt. Text: Sieber Bd. 2, S. 348

Engl. Text: State Papers Bd. 58, S. 986

Malloy, S. 53

Dyne, S. 444

\section{Einbürgerungsvertrag mit Württemberg}

mit. Protokoll vom gleichen Tage (Cahn, 1. Aufl., S. 404)

Prokl.: 7. 3. 1870

Amtl. Text: RegBl. 1872, S. 172

Statutes Bd. 16, S. 735

TS 375

Dt. Text: Sieber Bd. 2, S. 346

Bazille und Köstlin, 1902, S. 374 (Protokoll: S. 377)

Engl. Text: State Papers Bd. 58, S. 1176

Malloy, S. 1895

Dyne, S. 472

\section{Einbürgerungsvertrag mit Hessen-Darmstadt}

mit Protokoll vom gleichen Tage (Cahn, 1. Aufl., S. 406)

Prokl.: 31. 8. 1869

Amtl. Text: RegBl. 1869, S. 597

Statutes Bd. 16, S. 743

TS 171

Dt. Text: Sieber Bd. 2, S. 351

Engl. Text: State Papers Bd. 63, S. 887

Malloy, S. 949

Dyne, S. 463

16. 11. 1868 Einbürgerungsvertrag mit Belgien

(10.7.1869) Prokl.: 30. 7.1869

Amtl. Text: Statutes Bd. 16, S. 747

TS 24

Moniteur Belge Nr. 196 v. 15. 7. 1869

(Gesetz v. 10. 7. 1869) 
Engl. Text: State Papers, Bd. 58, S. 991

Malloy, S. 80

Dyne, S. 451

Hyatt, S. 1218

Franz. Text: Zeballos, Bd. 4, S. 416

Dt. Übers.: Sieber, Bd. 2, S. 355

Anwendung: Hackworth, S. 378-383

26. 5.1869

(14.6.1871)

Einbürgerungsvertrag mit Schweden und Norwegen

mit Zusatzprotokoll vom gleichen Tage. Der noch zur Zeit der Realunion geschlossene Vertrag gilt für Norwegen und Schweden noch heute.

Prokl.: 12. 1. 1872

Amtl. Text: Statutes, Bd. 17, S. 809

TS 350

Engl. Text: State Papers, Bd. 59, S. 272

Malloy, S. 1758

Hyatt, S. 1245

Dyne, S. 468 (Protokoll: S. 470)

Franz. Text: Zeballos, Bd. 4, S. 414 (Protokoll: S. 415)

Dt. Übers.: Sieber, Bd. 2, S. 361

Fälle: Perkins v. Elg (1939); Hansen v. Corson (1931);

in re Tobiassen (1929-32); vgl. dazu: Hackworth,

S. $392-398$

13. 5.1870

Einbürgerungsvertrag mit Großbritannien

(10. 8. 1870) Vorausgegangen war ein Protokoll Johnson-Stanley vom 9. 10. 1868

(Text: For.Rel. 1868 I, S. 359), dem der Vertrag grundsätzlich folgte. Dazu: Morrow, in: Am. Hist. Rev. 1933/4, S. 663-681. Kündigung Großbritanniens: 15. 12. 1953 (Cmd. 8757 und State Papers Bd. 160, S. 365), wirksam ab 15. 12. 1954, auch für SüdRhodesien und andere von Großbritannien außenpolitisch vertretene Territorien. Gleichzeitig Kündigung durch: Australien, Canada, Ceylon, Indien, Neuseeland, Pakistan, Südafrika. Für Irland Weitergeltung anzunehmen (s. Vertrag v. 21. 1. 1950).

Prokl.: 16. 9. 1870

Amtl. Text: Statutes, Bd. 16, S. 775

TS (US) 130

TS (UK) 9 von 1953 (Cmd. 8757)

Engl. Text: State Papers, Bd. 60, S. 36

Malloy, S. 691

Martens N. R. G., Bd. 20, S. 524

Dyne, S. 456

Hyatt, S. 1229

Franz. Text: Zeballos, Bd. 4, S. 411

Dt. Übers.: Sieber, Bd. 2, S. 359

Anwendung: Hackworth, S. 358-360 und Reynolds v.

Haskins (1925); U. S. v. Reid (1934); Ostby v.

Salmon (1929); Perkins v. Elg (1939); U. S. ex rel.

Guest v. Perkins (1936)

20.9.1870 Einbürgerungsvertrag mit Ơsterreich-Ungarn

(14. 7. 1871) Außer Kraft mit Kriegseintritt 1917

Prokl.: 1. 8. 1871

Amtl. Text: Statutes, Bd. 17, S. 833

TS 12

Dt. Text: Sieber, Bd. 2, S. 353

Engl. Text: State Papers, Bd. 60, S. 169

Malloy, S. 45

Martens, N. R. G., Bd. 20, S. 347 
Dyne, S. 441

Franz. Text: Zeballos, Bd. 4, S. 396

Anwendung: Hackworth, S. 377-378

6. 12.1870 Vertrag über Freundschaft, Handel und Konsularrechte mit Salvador

(11. 3. 1874) Außer Kraft am 30. 5. 1893 durch Kündigung Salvadors

StA/WdB: Art. 29 Abs. 1 und 3

Prokl.: 13. 3. 1874

Amtl. Text: Statutes, Bd. 18 III, S. 725

TS 310

Engl. Text: Malloy, S. 1551

State Papers, Bd. 67, S. 989

Martens, N.R.G. ${ }^{2}$, Bd. 1, S. 79

23. 2. 1871 Vertrag mit Großbritannien über Verzicht auf Einbürgerung

(4. 5. 1871) Prokl.: 5. 5. 1871

Amtl. Text: Statutes, Bd. 17, S. 841

TS 132

Engl. Text: Malloy, S. 698

Martens N. R. G., Bd. 20, S. 526

State Papers, Bd. 61, S. 38

Dyne, S. 458

26. 2. 1871 Handels- und Schiffahrtsvertrag mit Italien

(18.11.1871) Außer Kraft durch Krieg 1941

StA/WdB: Art. 3 Abs. 2

Prokl.: 23. 11. 1871

Amtl. Text: Statutes Bd. 17, S. 845

TS 177

Engl. Text: Malloy, S. 969

State Papers, Bd. 61, S. 88

Martens N.R.G. ${ }^{2}$, Bd. 1, S. 57

Fälle: Storti v. Massachusetts; Maiorano v. Baltimore \& Ohio Ry. Co.; Cantini v. Tillman; Tuleo v. Schuylkill Stone Co.

22. 12.1871 Freundschafts-, Handels- und Auslieferungsvertrag mit dem

(18.8.1873) Oranjefreistaat

Außer Kraft am 4. 1. 1895 durch Kündigung des Oranjefreistaats (eingegl. in Brit. Empire)

StA/WdB: Art. 2 Abs. 1

Prokl.: 23. 8. 1873

Amtl. Text: Statutes, Bd. 18 II, S. 580

und Bd. 18 III, S. 749

TS 265

Engl. Text: Malloy, S. 1312

State Papers, Bd. 63, S. 893

Martens, N.R.G.', Bd. 1, S. 69

6.5.1872 Einbürgerungsvertrag mit Ekuador

(6.11.1873) Außer Kraft: 25. 8. 1892 durch Kündigung Ecuadors

Prokl.: 24. 11. 1873

Amtl. Text: Statutes, Bd. 18 II, S. 197

TS 78

Engl. Text: Martens, N.R.G. ${ }^{2}$, Bd. 1, S. 93

Malloy, S. 434

Franz. Ubers.: Zeballos, Bd. 4, S. 426

20.7.1872 Einbürgerungsvertrag mit Dänemark

(14. 3. 1873) Prokl.: 15. 4. 1873

Amtl. Text: Statutes, Bd. 17, S. 941

TS 69

Engl. Text: State Papers, Bd. 63, S. 889 
Malloy, S. 384

Hyatt, S. 1224

Dyne, S. 453

Martens, N.R.G.'2, Bd. 1, S. 64

Dt. Übers.: Sieber, Bd. 2, S. 357

Anwendung: Hackworth, S. 373, 383-384

11. 8. 1874 Einbürgerungsvertrag mit der Türkei

Austausch der Ratifikationsurkunden: 22. 4. 1875, zusammen mit dem Auslieferungsvertrag v. 11. 8. 1874. Bei der Ratifikation beharrten die Türken in einer Note auf dem (vom US-Senat aber eigenmächtig abgeänderten) Originaltext, der einen automatischen StA-Verlust vorsah, während die USA das "shall“ in "may" umgewandelt hatten. Darauf erklärten die USA die Ratifikation für ungültig und der Vertrag wurde nicht proklamiert. Versuche, ihn doch in Kraft zu setzen, scheiterten 1889 und 1899 (dazu: AJIL 1931, S. 658 ff.). Bei den Verhandlungen über einen Vertrag vom 6. 8. 1923 war eine StA-Vorschrift vorgesehen, die aber dann nicht in den Text aufgenommen wurde: der Vertrag trat auch nie in Kraft (a. a. O., S. 667). Siehe auch noch AJIL 1908, S. 159.

Franz. Text: Noradounghian, Recueil des actes internationaux de l'Empire Ottoman, Bd. 3, Paris 1900, S. 368

14. 10. 1881 Handels- und Schiffahrtsvertrag mit Serbien

(15. 11. 1882) StA/WdB: Art. 4 Abs. 1

Prokl.: 27. 12. 1882

Amtl. Text: Statutes, Bd. 22, S. 963

TS 319

Engl. Text: Malloy, S. 1613

State Papers, Bd. 72, S. 714

Martens, N.R.G. ${ }^{2}$, Bd. 11, S. 744

2.10.1886 Freundschafts-, Handels- und Schiffahrtsvertrag mit Tonga

(1. 8. 1888) Außer Kraft am 28. 7. 1920, ausgenommen Art. 6

StA/WdB: Art. 9

Prokl.: 18. 9. 1888

Amtl. Text: Statutes, Bd. 25, S. 1440

TS 357

Engl. Text: Malloy, S. 1781

State Papers, Bd. 78, S. 778

24.1.1891 Freundschafts-, Handels- und Schiffahrtsvertrag mit dem Kongo

(2. 4. 1892) Ratifikationsaustausch am 2. 2. 1892; in Kraft mit Inkrafttreten der Brüsseler Sklaverei-Deklaration v. 2. 7. 1890 (Malloy, S. 1964)

für die USA

StA/WdB: Art. 3

Prokl.: 2. 4. 1892

Amtl. Text: Statutes, Bd. 27, S. 926

TS 60

Engl. Text: Malloy, S. 328

State Papers, Bd. 83, S. 371

Engl. und franz. Text: Martens, N.R.G.' ${ }^{2}$ Bd. 18, S. 577

22. 11.1894 Handels- und Schiffahrtsvertrag mit Japan

(17.7.1899) Außer Kraft mit Ablauf der zwölfjährigen Laufzeit am 17. 7. 1911

StA/WdB: Art. 1 Abs. 5

Prokl.: 21. 3. 1895

Amtl. Text: Statutes, Bd. 29, S. 848

TS 192

Engl. Text: Malloy, S. 1028

State Papers, Bd. 86, S. 524, 532

Martens, N.R.G. ${ }^{2}$, Bd. 34, S. 450

Fall: The Japanese Immigration Case 
10.12.1898 Friedensvertrag mit Spanien

(11. 4. 1899) StAB: Art. 9 und 11

Prokl.: 11. 4. 1899

Amtl. Text: Statutes, Bd. 30, S. 1754

TS 343

Engl. Text: State Papers, Bd. 90, S. 382

Martens, N.R.G. ${ }^{2}$, Bd. 32, S. 74

Malloy, S. 1690

Flournoy, S. 617 (Art. 9)

Fälle der sog. Insular Cases bei Dyne, S. 296-309; Fälle für

Puerto Rico und Philippinen a. a. O., S. 309-316.

Ferner s. Hackworth, S. 372 u. For. Rel. 1900, S. 894

29. 3. 1900 Übereinkommen (Protokoll) mit Spanien betr. Verlängerung der Optionsfrist in Art. 9 des Friedensvertrages, mit Wirkung für die Philippinen

Prokl.: 28. 4. 1900

Amtl. Text: Statutes, Bd. 31, S. 1881

TS 344

Engl. Text: State Papers, Bd. 92, S. 1128

Martens, N.R.G. ${ }^{2}$, Bd. 32, S. 293

Malloy, S. 1695

Hyatt, S. 1244

22. 3. 1902 Einbürgerungsvertrag mit Haiti

(19.3. 1904) Prokl.: 24. 3. 1904

Amtl. Text: Statutes Bd. 33, S. 2101

TS 432

Engl. Text: Malloy, S. 939

Flournoy, S. 681

Hyatt, S. 1231

Dyne, S. 461

Engl. u. franz. Text: State Papers, Bd. 95, S. 754

Martens, N.R.G.'2, Bd. 31, S. 291

Franz. Text: Sieber, Bd. 2, S. 362

Bourbousson, Traité gén. de la nationalité, 1931, S. 433

Chancy, Code des lois usuelles, 3. Aufl., Port-au-Prince, 1954, S. 115

3.7.1902 Vertrag über Freundschaft und allgemeine Beziehungen

(14. 4. 1903) mit Spanien $\quad$ StA/WdB: Art. 5 Abs. 1

Prokl.: 20. 4. 1903

Amtl. Text: Statutes, Bd. 33, S. 2105

TS 422

Engl. Text: Malloy, S. 1701

State Papers, Bd. 95, S. 807

Martens N.R.G. ${ }^{2}$, Bd. 31, S. 369

28. 2. 1903 Vertrag mit Haiti zur Verlängerung der Ratifikationsfrist

(19.3. 1904) für den Vertrag von 1902

Prokl.: 24. 3. 1904

Amtl. Text: Statutes, Bd. 33, S. 2157

TS 433

Engl. Text: Malloy, S. 941

Dyne, S. 463

Engl. u. franz. Text: State Papers, Bd. 96, S. 645

Martens, N.R.G. ${ }^{2}$, Bd. 31, S. 523

15. 10.1907 Einbürgerungsvertrag mit Peru

(23. 7. 1909) Gebilligt durch Res. Leg. Nr. 1074 vom 11. 3. 1909 (Peru) und vom US-Präsidenten am 9. 3. 1908

Prokl.: 2. 9. 1909 
Amtl. Text: Statutes, Bd. 36, S. 2181

TS 532

Engl. Text: State Papers, Bd. 101, S. 544

AJIL 1910 Suppl., S. 134

Malloy, S. 1449

Flournoy, S. 683

Hyatt, S. 1239

Engl. u. span. Text: Martens, N.R.G. ${ }^{3}$, Bd. 3, S. 233

Span. Text: Nieves Ayala, Los extranjeros, Lima 1950, S. 91

14. 3.1908 Einbürgerungsvertrag mit Salvador

(20.7.1908) Prokl.: 23. 7. 1908

Amtl. Text: Statutes, Bd. 35, S. 2038

TS 503

Engl. Text: State Papers, Bd. 102, S. 795

AJIL 1908 Suppl., S. 342

Malloy, S. 1570

Hyatt, S. 1242

Engl. u. span. Text: Martens, N.R.G. ${ }^{3}$, Bd. 3, S. 236

27. 4. 1908 Einbürgerungsvertrag mit Brasilien

(28. 2. 1910) Brasilien kündigte mit Note v. 13. 12. 1950, wirksam ab 14. 12. 1951

(State Papers, Bd. 158, S. 730)

Brasil. Dekret Nr. 2116 vom 8. 10. 1909

Prokl.: 2. 4. 1910

Amtl. Text: Statutes, Bd. 36, S. 2444

TS 547

Engl. Text: State Papers, Bd. 101, S. 920

AJIL 1910, Suppl., S. 262

Hyatt, S. 1219

Malloy, S. 2502

Engl. u. portugies. Text: Martens, N.R.G. ${ }^{3}$, Bd. 4, S. 250

7. 5. 1908 Einbürgerungsvertrag mit Portugal

(14. 11.1908) Prokl.: 14.12. 1908

Amtl. Text: Statutes, Bd. 35, S. 2082

TS 513

Engl. Text: AJIL 1909, Suppl., S. 159

Malloy, S. 1468

State Papers, Bd. 101, S. 1042

Hyatt, S. 1240

Engl. u. portugies. 'Text: Martens, N.R.G. ${ }^{3}$, Bd. 3, S. 239

Franz. Úbers.: Zeballos, Bd. 4, S. 417

Fälle: Hackworth, S. 365, 390-392

23. 6. 1908 Einbürgerungsvertrag mit Honduras

(16. 4. 1909) Prokl.: 8. 6. 1909

Amtl. Text: Statutes, Bd. 36, S. 2160

TS 525

Engl. Text: State Papers, Bd. 101, S. 1061

Malloy, S. 958

Hyatt, S. 1232

Engl. u. span. Text: Martens, N.R.G. ${ }^{3}$, Bd. 3, S. 242

Franz. Úbers.: Zeballos, Bd. 4, S. 427

10. 8. 1908 Einbürgerungsvertrag mit Uruguay

Gebilligt von Uruguay durch Gesetz Nr. 3435 v. 23. 3. 1909

(14. 5. 1909) Prokl.: 19.6.1909

Amtl. Text: Statutes, Bd. 36, S. 2165

TS 527 
Engl. Text: State Papers, Bd. 102, S. 895

AJIL 1909, Suppl., S. 284

Malloy, S. 1829

Engl. u. span. Text: Martens, N.R.G. ${ }^{3}$, Bd. 3, S. 245

Span. Text: Tratados y Convenios Internacionales, Bd. 1, 1960, S. 116

7. 12. 1908

(28. 3. 1912)

Einbürgerungsvertrag mit Nicaragua

Prokl.: 10. 5. 1912

Amtl. Text: Statutes, Bd. 37, S. 1560

TS 566

Engl. Text: State Papers, Bd. 102, S. 722

AJIL 1912, Suppl., S. 209

Malloy, S. 2738

Hyatt, S. 1237

Engl. u. span. Text: Martens, N.R.G. ${ }^{3}$, Bd. 6, S. 464

21. 2. 1911 Handels- und Freundschaftsvertrag mit Japan

(17.7. 1911) Außer Kraft durch Kriegsausbruch 1941

StA/WdB: Art. 1 Abs. 4

Prokl.: 5. 4. 1911

Amtl. Text: Statutes, Bd. 37, S. 1504

TS 558

Engl. Text: Malloy, S. 2712

State Papers, Bd. 105, S. 728

Martens N.R.G. ${ }^{3}$, Bd. 6, S. 729

10.6. 1911 Einbürgerungsvertrag mit Costarica

(9. 5. 1912) Prokl.: 6. 6. 1912

Amtl. Text: Statutes, Bd. 37, S. 1603

TS 570

Engl. Text: State Papers, Bd. 105, S. 878

Hyatt, S. 1222

A JIL 1912, Suppl., S. 242

Malloy, S. 2544

Engl. u. span. Text: Martens, N.R.G. ${ }^{3}$, Bd. 6, S. 468

Anwendung: Hackworth, S. 372-373

17. 6. 1911 Vertrag mit Nicaragua zur Verlängerung der Ratifikationsfrist

(28. 3. 1912) für den Vertrag von 1908

Prokl.: 10. 5. 1912

Amtl. Text: Statutes, Bd. 37, S. 1563

TS 567

Engl. Text: Malloy, S. 2739

State Papers, Bd. 105, S. 761

AJIL 1912, Suppl., S. 211

Engl. u. span. Text: Martens, N.R.G. ${ }^{3}$, Bd. 6, S. 467

4. 8. 1916 Vertrag mit Dänemark über Abtretung der westindischen Inseln

(17. 1. 1917) StAB: Art. 6

Prokl.: 25. 1. 1917

Amtl. Text: Statutes, Bd. 39, S. 1706

TS 629

Engl. Text: State Papers, Bd. 110, S. 843

Virgin Islands Code, Washington 1957, Bd. 1, S. XLV

Flournoy, S. 625 (Art. 6)

Malloy, S. 2558

Engl. u. dän. Text: Martens, N.R.G. ${ }^{3}$, Bd. 10, S. 357

3.6.1918 Verträge mit Großbritannien betr. Militärdienst von Engländern

(30.7.1918) bzw. Canadiern

Prokl.: 30. 7. 1918

Gebilligt durch britische Order in Council v. 4. 9. 1918

(State Papers, Bd. 111, S. 192) aufgrund des „The Military Service 
(Conventions with Allied States) Act, 1917“

Amtl. Text: Statutes, Bd. 40, S. 1620 und 1624

TS 633 und 634

Parl. Pap. Misc. Nr. 14 (1918)

Engl. Text: State Papers, Bd. 111, S. 568 (UK), 572 (Canada)

Malloy, S. 2650 bzw. 2654

Martens, N.R.G. ${ }^{3}$, Bd. 10, S. 395,400

Material: Hackworth, S. 360

24. 8. 1918 Vertrag mit Italien betr. Militärdienst

(12. 11. 1918) StAB: Art. 5

Prokl.: 18. 11. 1918

Amtl. Text: Statutes, Bd. 40, S. 1633

TS 637

Engl. Text: State Papers, Bd. 111, S. 763

Foreign Relations 1918, Suppl. 2, S. 726

Malloy, S. 2708

Engl. u. ital. Text: Martens, N.R.G. ${ }^{3}$, Bd. 10, S. 402

Trattati e Conv., Bd. 23, S. 519

17./30. 8. 1918 Vertrag mit Griechenland betr. Militärdienst

(12.11.1918) Prokl.: 18.11. 1918

Amtl. Text: Statutes, Bd. 40, S. 1637

TS 638

Engl. Text: State Papers, Bd. 111, S. 747

Malloy, S. 2663

Martens, N.R.G. ${ }^{3}$, Bd. 10, S. 406

Material: Hackworth, S. 360-361

3. 9.1918 Vertrag mit Frankreich betr. Militärdienst

(8.11.1918) Prokl.: 11.11.1918

Amtl. Text: Statutes, Bd. 40, S. 1629

TS 636

Engl. Text: State Papers, Bd. 111, S. 740

Malloy, S. 2592

Engl. u. franz. Text: Martens, N.R.G. ${ }^{3}$, Bd. 10, S. 409

16. 12. 1920 Vertrag mit Thailand über die Revision früherer Verträge

(1. 9. 1921) (Freundschaftsvertrag usw.)

Außer Kraft am 1.10.1938

StA/WdB: Art. 1 Abs. 4

Prokl.: 12. 10. 1921

Amtl. Text: Statutes, Bd. 42, S. 1928

TS 655

LNTS Bd. 6, S. 291

Engl. Text: Malloy, S. 2829

State Papers, Bd. 113, S. 1168

Martens, N.R.G. ${ }^{3}$, Bd. 15, S. 828

25.8.1921 Friedensvertrag mit Deutschland

(11. 11. 1921) StAB: Art. 2, Z. 1 des Vertrages erklärt u. a. Kap. X des Versailler Vertrages für anwendbar, womit auch die StAB des Art. 278

Versailler Vertrag gilt

Prokl.: 14. 11. 1921

Gebilligt durch dt. Gesetz v. 20. 10. 1921

Amtl. Text: Statutes, Bd. 42, S. 1939

TS 658

RGBl. 1921, S. 1317

LNTS, Bd. 12, S. 191

Engl. Text: Malloy, S. 2596

Engl. u. deutscher Text: Martens, N.R.G. ${ }^{3}$, Bd. 11, S. 917 
23. 11. 1923 Einbürgerungsvertrag mit Bulgarien

(5. 4. 1924) Außer Kraft mit Kriegserklärung: 13.12.1941 (Bulgarien), 18. 7.1942 (USA)

Wieder in Kraft: 8. 3. 1948 (Statutes, Bd. 61, S. 1915; TIAS 1650;

UNTS, Bd. 29, S. 101) durch U.S-Note vom gleichen Tage, gemäß Art. 8 des Friedensvertrages v. 10. 2. 1947 (UNTS, Bd. 41, S. 21). In Wtb. des Völkerrechts, Bd. 1, 1960, S. 153, fälschlich als außer Kraft angeführt. In Treaties in Force 1965, S. 20, richtig als geltend angeführt

Prokl.: 6. 5. 1924

Amtl. Text: Statutes, Bd. 43, S. 1759

TS 684

LNTS, Bd. 25, S. 238

Engl. Text: State Papers, Bd. 118, S. 1067

Malloy, S. 3972

Hyatt, S. 1221

Martens, N.R.G. ${ }^{3}$, Bd. 13, S. 245

Anwendung: Hackworth, S. 403-407

8. 12. 1923 Handels-, Schiffahrts- und Konsular-Vertrag mit Deutschland

(14. 10.1925) Der durch den II. Weltkrieg außer Kraft getretene Vertrag wurde durch Übereinkommen v. 3. 6. 1953 (UNTS, Bd. 253, S. 89; State Papers, Bd. 160, S. 686) ab 22. 10. 1954 wieder wirksam (BGBl 1954 II, S. 721 u. 1051), jedoch war Art. 6 durch Kündigung der USA schon am 2. 6. 1954 außer Kraft getreten, nämlich durch Notenwechsel vom 2. 6. 1953 (TIAS 2972; UST, Bd. 5, S. 827; State Papers, Bd. 160, S. 684)

StAB: Art. 6 (Wehrdienst)

Prokl.: 14. 10. 1925

Amtl. Text: Statutes, Bd. 44, S. 2132

TS 725

RGBl. 1925 II, S. 795

LNTS, Bd. 52, S. 133

Engl. Text: State Papers, Bd. 122, S. 807

Malloy, S. 4191

Engl. u. deutscher Text: Martens, N.R.G. ${ }^{3}$, Bd. 17, S. 353

24. 6. 1925 Handels-, Schiffahrts- und Konsular-Vertrag mit Ungarn

(4. 9. 1926) Zunächst außer Kraft durch Kriegserklärungen Ungarns v. 13.12. 1941 u. der USA v. 18. 7. 1942, gemäß Art. 10 des Friedensvertrages vom 10. 2. 1947 (UNTS, Bd. 41, S. 135) durch Note der USA vom 9. 3. 1948 (UNTS, Bd. 183, S. 3) wieder in Kraft gesetzt, aber durch Kündigung der USA vom 5. 7. 1951 (UNTS, Bd. 141, S. 412) außer Kraft am 5. 7. 1952

StAB: Art. 6 (Wehrdienst)

Prokl.: 4. 10. 1926

Amtl. Text: Statutes, Bd. 44, S. 2441

TS 748

LNTS, Bd. 58, S. 111

Engl. Text: State Papers, Bd. 124, S. 899

Malloy, S. 4318

Martens, N.R.G. ${ }^{3}$, Bd. 18, S. 136

23. 12. 1925 Freundschafts-, Handels- und Konsularvertrag mit Estland

(22. 5. 1926) StAB: Art. 6 (Wehrdienst)

Prokl.: 25. 5. 1926

Amtl. Text: Statutes, Bd. 44, S. 2379

TS 736

LNTS, Bd. 50, S. 13

Engl. Text: State Papers, Bd. 122, S. 478

Malloy, S. 4105

Martens, N.R.G. ${ }^{3}$, Bd. 18, S. 275 
22. 2. 1926 Freundschafts-, Handels- und Konsularvertrag mit Salvador

(5. 9. 1930) Außer Kraft durch Kündigung seitens USA v. 7. 2. 1957, wirksam 8. 2. 1958 (Whiteman, Bd. 8, S. 564)

StAB: Art. 6 (Wehrdienst)

Prokl.: 8. 9. 1930

Amtl. Text: Statutes, Bd. 46, S. 2817

TS 827

LNTS, Bd. 134, S. 207

Engl. Text: State Papers, Bd. 133, S. 833

Malloy, S. 4615

Engl. u. span. 'Text: Martens, N.R.G. ${ }^{3}$, Bd. 26, S. 533

7.12. 1927 Freundschafts-, Handels- und Konsularvertrag mit Honduras

(19.7.1928) StAB: Art. 6 (Wehrdienst)

Prokl.: 23. 7. 1928

Amtl. Text: Statutes, Bd. 46, S. 2618

TS 764

LNTS, Bd. 87, S. 421

Engl. Text: State Papers, Bd. 127, S. 723

Malloy, S. 4306

Engl. u. span. Text: Martens, N.R.G. ${ }^{3}$, Bd. 21, S. 647

Über Wehrdienst v. Doppelstaatern: Hackworth, S. 361-364

20. 4. 1928 Freundschafts-, Handels- und Konsularvertrag mit Lettland

(25.7.1928) StAB: Art. 6 (Wehrdienst)

Prokl.: 25. 7. 1928

Amtl. Text: Statutes, Bd. 45, S. 2641

TS 765

LNTS, Bd. 80, S. 35

Engl. Text: State Papers, Bd. 129, S. 827

Malloy, S. 4400

Martens, N.R.G. ${ }^{3}$, Bd. 31, S. 198

5. 6. 1928 Freundschafts-, Handels- und Konsularvertrag mit Norwegen

(13. 9. 1932) StAB: Art. 6 (Wehrdienst)

Prokl.: 15. 9. 1932

Amtl. Text: Statutes, Bd. 47, S. 2135

TS 852

LNTS, Bd. 134, S. 81

Engl. Text: State Papers, Bd. 131, S. 588

Malloy, S. 4527

Engl. u. norweg. Text: Martens, N.R.G. ${ }^{3}$, Bd. 31, S. 293

19.6. 1928 Freundschafts-, Handels- und Konsularvertrag mit Österreich

(27. 5. 1931) Gilt noch heute

StAB: Art. 6 (Wehrdienst)

Prokl.: 28. 5. 1931

Amtl. Text: Statutes, Bd. 47, S. 1876

TS 838

LNTS, Bd. 118, S. 241

Dt. Text: öBGBl. 1931, S. 873 (und engl.)

Engl. Text: Malloy, S. 3930

Engl. u. deutscher Text: Martens, N.R.G. ${ }^{3}$, Bd. 25, S. 108

16.7.1928 Einbürgerungsvertrag mit der CSR

(14.11.1929) Gilt noch heute unverändert

Prokl.: 14. 11. 1929

Amtl. Text: Statutes, Bd. 46, S. 2424

TS 804

LNTS, Bd. 99, S. 301

Engl. Text: State Papers, Bd. 129, S. 459

Malloy, S. 4063 
Hyatt, S. 1223

Martens, N.R.G. ${ }^{3}$, Bd. 21, S. 765

1. 11.1930

(11. 2. 1931)

5. 4. 1932

(22.7.1935)

31. 1.1933

(20.2.1935)

18. 10. 1937

(20.7.1938)

Abkommen mit Norwegen über Militärdienst von Doppelstaatern

Prokl.: 12. 2. 1931

Amtl. Text: Statutes, Bd. 46, S. 2904

TS 832

LNTS, Bd. 112, S. 399

Engl. Text: State Papers, Bd. 133, S. 783

Malloy, S. 4542

Hyatt, S. 1238

Engl. u. norweg. Text: Martens, N.R.G. ${ }^{3}$, Bd. 24, S. 448

Fälle: Hackworth, S. 408-410; A JIL 1931, S. 119 f.

\section{Einbürgerungsvertrag mit Albanien}

Durch Entschließung Nr. 3 des Antifaschistischen Rates der Nationalen Befreiungsfront, die noch vor der Machtübernahme im November 1944 erging, wurden alle Vorkriegsverträge einschließlich derer über StA für ungültig erklärt (Gazeta Zyrtare Nr. 1 v. 1944). Die Akte des Rates wurden im Jan. 1946 von der Konstituante in toto bestätigt (G.Z. Nr. 12 v. 1946): So Angaben ohne Kommentar in Osteuropa-Recht 1967, S. 245/6. Der Vertrag wird von den USA als weiterhin geltend betrachtet und in Treaties in Force stets aufgeführt.

Prokl.: 29. 7. 1935

Amtl. Text: Statutes, Bd. 49, S. 3241

TS 892

LNTS, Bd. 162, S. 31

Engl. Text: State Papers, Bd. 135, S. 803

Malloy, S. 3922

Hyatt, S. 1217

Martens, N.R.G. ${ }^{3}$, Bd. 31, S. 398

Anwendung: Hackworth, S. 403-407

Abkommen mit Schweden über Militärdienst von Doppelstaatern

Prokl.: 20. 5. 1935

Amtl. Text: Statutes, Bd. 49, S. 3195

TS 890

LNTS, Bd. 159, S. 261

Engl. Text: State Papers, Bd. 136, S. 831

Malloy, S. 4656

Hyatt, S. 1245

Engl. u. schwed. Text: Martens, N.R.G. ${ }^{3}$, Bd. 31, S. 401

Material bei Hack worth, S. 358, 410 f.

Abkommen mit Litauen über Militärdienst der Doppelstaater

Gebilligt durch lit. Präs. am 30. 12. 1937

Prokl.: 15. 8. 1938

Amtl. Text: Statutes, Bd. 53, S. 1569

TS 936

V.Z. 1938, Nr. 623, Pos. 4441

LNTS, Bd. 191, S. 351

Dt. Text: ABl. Memel 1938, S. 1079

Engl. Text: Hyatt, S. 1234

Martens, N.R.G. ${ }^{3}$, Bd. 36, S. 73

Material bei Hackworth, S. 411-412

11.11.1937 Abkommen mit der Schweiz über Militärdienst der Doppelstaater

(7.12.1938) Ein Entwurf eines umfassenden StA-Vertrages, den die USA 1931 vorlegten, wurde von der Schweiz nicht gebilligt (Hackworth, S. 412) Prokl.: 13. 12. 1938 
Amtl. Text: Statutes, Bd. 53, S. 1791

TS 943

LNTS, Bd. 193, S. 181

Dt. Text: Ber. Slg. Bd. 11, Bern 1953, S. 588

Engl. Text: State Papers, Bd. 141, S. 1259

Hyatt, S. 1248

Engl. u. franz. Text: Martens, N.R.G. ${ }^{3}$, Bd. 37, S. 39

Material bei Hackworth, S. 366, 412 f.

13.11.1937 Freundschafts-, Handels- und Schiffahrtsvẹrtrag

(1. 10.1938) mit Thailand (Siam)

StA/WdB: Art. 1 Abs. 4

Amtl. Text: Statutes, Bd. 53, S. 1731

TS 940

LNTS, Bd. 192, S. 248

Engl. Text: State Papers, Bd. 141, S. 1214

8. 8. 1938 Freundschafts-, Handels- und Konsularvertrag mit Liberia

(21. 11. 1939) StAB: Art. 6 (Wehrdienst, mit 2. Absatz)

Amtl. Text: Statutes, Bd. 54, S. 1739

TS 956

LNTS, Bd. 201, S. 163

Engl. Text: State Papers, Bd. 142, S. 705

27. 1. 1939 Abkommen mit Finnland betr. Militärdienst der Doppelstaater

(3. 10. 1939) $\mathrm{Da}$ im Zweiten Weltkrieg kein Kriegszustand zwischen Finnland und den USA bestand, gilt der Vertrag unverändert weiter

Prokl.: 7. 10. 1939

Amtl. Text: Statutes, Bd. 54, S. 1712

TS 953

LNTS, Bd. 201, S. 197

Engl. Text: State Papers, Bd. 143, S. 473

Hyatt, S. 1227

Martens, N.R.G. ${ }^{3}$, Bd. 37, S. 701

Anwendung: Hackworth, S. 413

18. 3. 1942 Notenwechsel betr. Übertritt v. US-Bürgern und ehemaligen US-

(20. 3. 1942) Bürgern aus kanadischen in amerikanische Streitkräfte und umgekehrt

v. 18. u. 21. 3. 1942

Amtl. Text: UNTS, Bd. 105, S. 169

Engl. Text: AJIL 1942, Doc. S. 156

30.3.1942 Notenwechsel mit Canada betr. Anwendung des US-Wehrgesetzes

(6. 4. 1942) auf Canadier und umgekehrt

v. 30. 3., 6. 4., 8. 4. 1942

Amtl. Text: EAS 249

Statutes, Bd. 56, S. 1477

UNTS, Bd. 105, S. 179

Engl. Text: AJIL 1942, Doc. S. 158

30. 3. 1942 Notenwechsel betr. Wehrdienst mit Großbritannien

(30. 4. 1942) v. 30. 3., 29. 4., 9. 6., 30. 9. 1942

Amtl. Text: EAS 307

Statutes, Bd. 56, S. 1906

UNTS, Bd. 13, S. 169

Engl. Text: State Papers, Bd. 144, S. 1054

30. 3. 1942 Notenwechsel betr. Wehrdienst mit Indien

(27. 5. 1942) v. 30. 3., 25. 5., 3. 7., 3. 9. 1942

Amtl. Text: EAS 308

Statutes, Bd. 56, S. 1912

UNTS, Bd. 13, S. 185 
30. 3. 1942 Notenwechsel betr. Wehrdienst mit Polen

(27. 1. 1943) v. 30. 3., 14. 12. 1942, 26. 1., 25. 2. 1943

Amtl. Text: EAS 320

Statutes, Bd. 57, S. 954

UNTS, Bd. 13, S. 395

31. 3. 1942 Notenwechsel betr. Wehrdienst mit Jugoslawien

(18. 5. 1942) v. 31.3., 14. 5., 25.6., 30.9. 1942

Amtl. Text: EAS 309

Statutes, Bd. 56, S. 1917

UNTS Bd. 13, S. 199

31. 3. 1942 Notenwechsel betr. Wehrdienst mit Südafrika

(11. 6. 1942) v. 31. 3., 9. 6., 12. 8., 7. 10., 31. 10. 1942

Amtl. Text: EAS 310

Statutes, Bd. 56, S. 1921

UNTS, Bd. 105, S. 269

31. 3. 1942 Notenwechsel betr. Wehrdienst mit Neuseeland

(2.7. 1942)

v. 31. 1., 1. 7., 15. 8., 30. 9. 1942

Amtl. Text: EAS 305

Statutes, Bd. 56, S. 1896

UNTS, Bd. 13, S. 139

31. 3. 1942 Notenwechsel betr. Wehrdienst mit den Niederlanden

(8. 7. 1942) v. 31. 3., 2. 7., 24. 9., 30. 9. 1942

Amtl. Text: EAS 306

Statutes, Bd. 56, S. 1900

UNTS, Bd. 13, S. 151

31. 3. 1942 Notenwechsel betr. Wehrdienst mit Australien

(18.7.1942) v. 31. 3., 17.7., 16. 9., 30.9. 1942

Amtl. Text: EAS 303

Statutes, Bd. 56, S. 1884

UNTS, Bd. 13, S. 125

31. 3. 1942 Notenwechsel betr. Wehrdienst mit Belgien

(4.8.1942) v. 31.3., 31.7., 10.10., 16.10. 1942

Amtl. Text: EAS 304

Statutes, Bd. 56, S. 1889

UNTS, Bd. 13, S. 211

31. 3. 1942 Notenwechsel betr. Wehrdienst mit Norwegen

(24. 12.1942) v. 31. 3., 6. 10., 23. 12. 1942, 16.1. 1943

Amtl. Text: EAS 319

Statutes, Bd. 57, S. 949

UNTS, Bd. 13, S. 335

31. 3. 1942 Notenwechsel betr. Wehrdienst mit Griechenland

(2. 3. 1943) v. 31.3.1942, 8. 2., 2. 3., 16. 3. 1943

Amtl. Text: EAS 322

Statutes, Bd. 57, S. 968

UNTS, Bd. 105, S. 227

3. 4. 1942 Notenwechsel betr. Wehrdienst mit der Tschechoslowakei

(29. 9.1943) v. 3. 4. 1942, 29.9., 21.10.1943

Amtl. Text: EAS 341

Statutes, Bd. 57, S. 1070

UNTS, Bd. 29, S. 369

6.11. 1942 Notenwechsel betr. Wehrdienst mit Cuba

(11.1.1943) v. 6.11.1942, 9.1., 1. 2. 1943

Amtl. Text: EAS 321 
Statutes, Bd. 57, S. 960

UNTS, Bd. 13, S. 379

22. 1. $1943 \quad$ Notenwechsel betr. Wehrdienst mit Mexico

(22. 1. 1943) Beide Noten v. 22. 1. 1943

Amtl. Text: EAS 323

Statutes, Bd. 57, S. 973

UNTS, Bd. 105, S. 259

Engl. Text: State Papers, Bd. 145, S. 309

23. 1. 1943 Notenwechsel betr. Wehrdienst mit Brasilien

(30. 4. 1943) v. 23.1., 28. 4., 24. 5. 1943

Amtl. Text: EAS 327

Statutes, Bd. 57, S. 994

UNTS, Bd. 28, S. 385

3. 4. 1943 Notenwechsel betr. Wehrdienst mit Salvador

(15. 5. 1943) v. 3. 4., 14. 5., 31. 5. 1943

Amtl. Text: EAS 325

Statutes, Bd. 57, S. 982

UNTS, Bd. 105, S. 205

6.11.1943 Notenwechsel betr. Wehrdienst mit China

(13.6.1944) v. 6.11.1943,11.5., 13.6.1944

Amtl. Text: EAS 426

Statutes, Bd. 58, S. 1442

UNTS, Bd. 107, S. 43

27. 1. 1944 Notenwechsel betr. Wehrdienst mit Columbien

(27.1.1944) v. 27.1., 12. 2. 1944

Amtl. Text: EAS 407

Statutes, Bd. 58, S. 1296

UNTS, Bd. 109, S. 287

2. 4. 1945 Notenwechsel betr. Wehrdienst mit Ecuador

(5. 4. 1945) v. 2. 4. und 5. 4.1945

Amtl. Text: EAS 475

Statutes, Bd. 59, S. 1598

UNTS, Bd. 121, S. 265

10.5.1945 Notenwechsel betr. Wehrdienst mit Venezuela

(11.5.1945) v. 10. und 11.5.1945

Amtl. Text: EAS 476

Statutes, Bd. 59, S. 1602

UNTS, Bd. 121, S. 273

23. 5. 1945 Notenwechsel betr. Wehrdienst mit Peru

(12.6.1945) v. 23. 5., 12.6. 1945

Amtl. Text: EAS 477

Statutes, Bd. 59, S. 1606

UNTS, Bd. 121, S. 283

7.6. 1945 Notenwechsel betr. Wehrdienst mit Chile

(11.6.1945) v. 7. und 11.6.1945

Amtl. Text: EAS 478

Statutes, Bd. 59, S. 1610

UNTS, Bd. 121, S. 291

4.11.1946 Freundschafts-, Handels- und Schiffahrtsvertrag mit China

(30. 11. 1948) Gilt heute für Taiwan (Republik China)

StAB: Art. 14 (Militärdienst, mit 2. und 3. Absatz)

Amtl. Text: Statutes, Bd. 63, S. 1299

TIAS 1871 
UNTS, Bd. 25, S. 69

Engl. Text: State Papers, Bd. 146, S. 686

16. 2.1

8. 3.1947

2. 4. 1947

(18.7. 1947)

Notenwechsel mit Polen über Errichtung einer Mixed Nationality

Commission

Aufgehoben durch Notenwechsel v. 21. 5./14. 6. 1948

(Dep.St.Bull. Nr. 467 v. 13. 6. 1948, S. 781).

Whiteman, S. 73, druckt nur den Notenwechsel von 1948 ab.

Text: nicht veröffentlicht

(

Treuhandabkommen mit der UNO über die ehemals japanischen Südsee-Inseln

StAB: Art. 11 (Pflicht zur StA-Regelung, ausgeführt durch Code v. 22. 12. 1952, Sec. 660-662)

Gebilligt von USA durch Joint Resolution v. 18. 7. 1947, c. 271

Amtl. Text: Statutes, Bd. 61 III, S. 3301

TIAS 1665

UNTS, Bd. 8, S. 189

Engl. Text: State Papers, Bd. 148, S. 513

Naval War College 1946/47, S. 146

Doc. int. aff. 1947/8, S. 843

Franz. Text: Notes et Etudes, Nr. 545

2. 2. 1948 Freundschafts-, Handels- und Schiffahrtsvertrag mit Italien

(26.7.1949) StAB: Art. 13 (Wehrdienst)

Gebilligt durch italienisches Gesetz Nr. 383 v. 8. 6. 1949

Prokl.: 5. 8. 1949

Amtl. Text: Statutes, Bd. 63, S. 2255

TIAS 1965

G. U. v. 12. 7. 1949, Nr. 157 (Suppl.)

UNTS, Bd. 79, S. 171

Engl. Text: State Papers, Bd. 152, S. 569

Bettoni, L'italiano nel mondo, Bd. 2, 1955, S. 896

Ital. Text: Annali di dir. int. 1949, S. 515

Material: Hackworth, S. 361, 373-77; SGS, Bd. 27

S. 465-467; Barone, in: Fordham, L. R. 1954, S. 243-295

25. 2. 1948 Notenwechsel mit Frankreich betr. Militärdienst der Doppelstaater

(25. 2. 1948) Amtl. Text: Statutes, Bd. 62, S. 1950

TIAS 1756

UNTS, Bd. 67, S. 33

J. O. v. 23. 4. 1948, S. 3957 (Dekret v. 16. 4. 1948)

Material: Hackworth, S. 358-360

22.12.1948 Zweites Übereinkommen mit Frankreich betr. Militärdienst der

(22.12.1948) Doppelstaater

Amtl. Text: Statutes, Bd. 62, S. 3621

TIAS 1876

UNTS, Bd. 67, S. 38

J. O. v. 4. 3. 1949 , S. 2340 (Dekret Nr. 290 v. 2. 3. 49)

Franz. Text: Rev. int. fr. dr. gens 1948, S. 127

Dalloz, B. L. 1949, S. 355

21. 1. 1950 Freundschafts-, Handels- und Schiffahrtsvertrag mit Irland

(14. 9. 1950) (nebst Protokoll vom selben Tage)

StAB: Art. 3 Abs. 2 (Wehrdienst)

Prokl.: 15. 12. 1950

Amtl. Text: UST, Bd. 1, S. 785, 803

TIAS 2155

TS (Irland) 1950, Nr. 7

UNTS, Bd. 206, S. 269

Engl. Text: State Papers, Bd. 157, S. 499 
23./25. 3. 1950 Notenwechsel mit Jugoslawien betr. Visa für amerikanische Staats(1. 4.1950) angehörige jugoslawischer Abstammung, die auch von Jugoslawien als Staatsangehörige beansprucht werden

Amtl. Text: TIAS 2087

UST, Bd. 1, S. 471

UNTS, Bd. 98, S. 195

11. 8. 1951 Notenwechsel über landwirtschaftliche Wanderarbeiter mit Mexiko

(11. 8. 1951) Beide Noten v. 11. 8. 1951. Ersetzt frühere Vereinbarungen v. 1. 8. 1949 u. 9. 3. 1951 (UST, Bd. 2, S. 1917; TIAS 2328)

StA/WdB: Art. 34

Amtl. Text: UST, Bd. 2, S. 1940

TIAS 2331

18. 11./ Notenwechsel mit Frankreich zur Ergänzung des Vertrages

31. 12. 1952 v. 22. 12. 1948

(31. 12. 1952/ Amtl. Text: UST, Bd. 3, S. 5345

22. 12. 1950) TIAS 2741

UNTS, Bd. 185, S. 396 


\section{ABKÜRZUNGEN}
AJIL
American Journal of International Law
Cahn, W.
Reichs- u. StAG v. 22. 7. 1913, 4 Auflagen, Berlin
Dyne, F. von
A treatise on the law of naturalization of the U.S.
Washington 1907
EAS
Executive Agreements Series
For.Rel.
Foreign Relations (United States)
Flournoy (-Hudson) A Collection of nationality laws, New York 1929
Hackworth, G. H. Digest of international law, Bd. 3, Washington 1942
Hyatt, C. B. u. a. Laws applicable to immigration and nationality Washington 1954
LNTS League of Nations Treaty Series
Malloy, W. M. u. a. Treaties, Conventions, International Acts ... Washington 1910-1938, 4 Bde.
Miller, $\mathrm{H}$.
Treaties and other International Acts of the United States of America, Bde. 2-7, Washington 1931-1948
Rabl, K. Das Recht auf die Heimat, Bd. 1, München 1958
SGS
Sammlung geltender Staatsangehörigkeitsgesetze
Sieber, J.
Das Staatsbürgerrecht, Bern 1907, 2 Bde.
StAB
Staatsangehörigkeitsbestimmung
TIAS
Treaties and other International Acts Series
UNTS
United Nations Treaty Series
TS
Treaty Series
UST
United States Treaties and Other International Agreements
WdB
Wehrdienstbestimmung
Whiteman, M. Digest of International Law, Bd. 8, Washington 1967
Zeballos, E.-S. La nationalité, Paris 1914-1919, 5 Bde. 